\title{
DNA replication in progenitor cells and epithelial regeneration after lung injury requires the oncoprotein MDM2
}

\author{
Shilpa Singh, ${ }^{1,2}$ Catherine A. Vaughan, ${ }^{1,2}$ Christopher Rabender, ${ }^{2,3}$ Ross Mikkelsen, ${ }^{2,3}$ Sumitra Deb, ${ }^{1,2}$ \\ and Swati Palit Deb ${ }^{1,2}$ \\ 'Department of Biochemistry and Molecular Biology, ${ }^{2}$ VCU Massey Cancer Center, and ${ }^{3}$ Department of Radiation Oncology, \\ Virginia Commonwealth, University, Richmond, Virginia, USA.
}

\begin{abstract}
Depletion of epithelial cells after lung injury prompts proliferation and epithelial mesenchymal transition (EMT) of progenitor cells, and this repopulates the lost epithelial layer. To investigate the cell proliferative function of human oncoprotein MDM2, we generated mouse models targeting human MDM2 expression in either lung Club or alveolar cells after doxycycline treatment. We report that MDM2 expression in lung Club or alveolar cells activates DNA replication specifically in lung progenitor cells only after chemical- or radiation-induced lung injury, irrespective of their p53 status. Activation of DNA replication by MDM2 triggered by injury leads to proliferation of lung progenitor cells and restoration of the lost epithelial layers. Mouse lung with no Mdm2 allele loses its ability to replicate DNA, whereas loss of $1 \mathrm{Mdm} 2$ allele compromises this function, demonstrating the requirement of endogenous MDM2. We show that the $p 53$-independent ability of MDM2 to activate Akt signaling is essential for initiating DNA replication in lung progenitor cells. Furthermore, MDM2 activates the Notch signaling pathway and expression of EMT markers, indicative of epithelial regeneration. This is the first report to our knowledge demonstrating a direct p53-independent participation of MDM2 in progenitor cell proliferation and epithelial repair after lung injury, distinct from a p53-degrading antiapoptotic effect preventing injury.
\end{abstract}

Conflict of interest: The authors have declared that no conflict of interest exists.

Copyright: (c) 2019, American Society for Clinical Investigation.

Submitted: February 18, 2019 Accepted: September 5, 2019 Published: October 17, 2019

Reference information: /CI Insight. 2019;4(20):e128194.

https://doi.org/10.1172/jici. insight.128194.

\section{Introduction}

Amplification or overexpression of the human homologue of the $m d m 2$ gene has been implicated in human cancers with or without p53 mutation (1-4). Moreover, a single nucleotide polymorphism (snp) at bp 309 of the MDM2 promoter leads to MDM2 overexpression $(5,6)$. Both of these genetic alterations, gene amplification and snp at 309, have been found in cancerous and normal lung tissues (7-10). These reports suggest that MDM2 overexpression could be one of the early events mediating proliferative effects in the lung.

The conventional paradigm ascribes the cell proliferative functions of MDM2 to its ability to destabilize the tumor suppressor p53. MDM2 interacts with WT p53 and ubiquitinates and targets the tumor suppressor for degradation $(1,11)$. While studies in animal models suggest an essential role of MDM2 in development through its ability to degrade and, thus, control growth-suppressing and apoptotic function of WT p53 $(12,13)$, consequences of MDM2 overexpression in animal models have been context dependent. Transgenic mice overexpressing MDM2 show tumor formation, although at a slower rate than p53-null mice (14). Although targeted overexpression of MDM2 in lactating mammary gland of mice prevents normal development or morphogenesis of mammary gland, it increases frequency of polyploid cells (15). MDM2 expression in the basal layer of epidermis at the embryonic stage generates hyperplasia and premalignant lesions (16); in wing and eye of drosophila, it induces apoptosis (17). The role of MDM2 in the maintenance of nephron progenitor cells during organogenesis has been ascribed to its E3 ligase function balancing p53 levels $(18,19)$. A recent study has reported that MDM2 prevents differentiation of cultured mesenchymal stem cells independently of p53 but promotes induced pluripotent stem cells (iPSC) in cultured mouse embryonic fibroblasts and clonogenic survival of cancer cells utilizing its ability of ubiquitination (20). These reports suggest that MDM2 participates in iPSC, and its overexpression may facilitate cell proliferative events in a context-dependent manner. However, the trigger or steps of the proliferative events in the complex organs remain unknown to date. 
Although MDM2 is frequently overexpressed in human lung cancers with WT or mutant p53 (2, 21, 22), the consequence of MDM2 overexpression in normal adult lung has not been investigated, and there is no existing mouse model to determine the cell-proliferative effects of MDM2 in adult lung. Lung is a highly quiescent organ with regenerative potential. Depletion of epithelial cells after lung injury activates proliferation of progenitor cells, which subsequently undergo epithelial mesenchymal transition (EMT) to repopulate the lost epithelial layer (23-25). Although crosstalk of several growth factors has been implicated in reepithelialization after lung injury (26), the mechanisms required for progenitor cell proliferation and injury repair are largely unknown. Pulmonary diseases induced by injury have often been associated with lung cancer $(27,28)$. The context-dependent cell proliferative properties of MDM2 overexpression led us to investigate whether injury could be one of the triggers to initiate cell-proliferative effects of MDM2 in the lung, thus mediating epithelial cell repopulation after lung injury.

Since biological functions of mouse or human MDM2 do not show strict species specificity (17, 29, 30), we investigated the cell-proliferative functions of human MDM2 using inducible mouse models. Thus, we have generated mouse models steering controlled lung-specific expression of human MDM2 from a doxycycline-inducible (Dox-inducible) Club cell secretory protein (CCSP) or surfactant protein C (SPC) promoter, in the context WT or mutant p53 in adult mice. Our results revealed the ability of MDM2 to induce DNA replication and proliferation of lung progenitor cells only after lung injury, leading to EMT and accelerated epithelial regeneration. This function of MDM2 did not require WT p53. Furthermore, $\mathrm{p} 53^{-/-}: \mathrm{Mdm} 2^{-/-}$mice lost the ability of progenitor cell proliferation, whereas $\mathrm{p} 53^{+/-}: \mathrm{Mdm} 2^{+/-}$mice displayed compromised ability of epithelial regeneration after lung injury, implicating the requirement of MDM2 in lung injury repair in normal adult animals. MDM2 also induced a p53-independent injury signaling pathway, and this function was essential for progenitor cell proliferation by MDM2. These observations imply that MDM2 overexpression may induce progenitor cell proliferation and accelerated reepithelialization in the aftermath of lung injury. Thus, pathological effects of enhanced and deregulated repair (27) could, in principle, be prevented by inhibiting MDM2-induced signaling pathway.

\section{Results}

Lung-specific induction of MDM2 expression in mice leads to expression of interphase markers but not DNA replication. To determine the consequence of elevated MDM2 expression in the lungs of adult mice, pTREtightMDM2 (a plasmid expressing MDM2 from CMV promoter controlled by tetracycline response element) transgenic mouse lines were generated (Figure 1A). The transgenic mouse line tightMDM2 was then generated by crossbreeding pTREtightMDM2 mice with CCSPrtTA transgenic mice (31), capable of expressing a reverse transactivator, rtTA, from CCSP promoter in response to Dox treatment. When treated with Dox, tightMDM2 mouse lines express the transactivator rtTA specifically in CCSP-expressing lung cells and activates TRE to induce MDM2 expression. CCSPrtTA (referred in the paper as rtTA) mice, which lacks mdm2 transgene, was used as control. Dox-induced MDM2 expression was confirmed at RNA (Figure 1B) and protein levels (Figure 1C) by quantitative PCR (qPCR) and immunoblot analysis, respectively. Dox treatment for 3 months resulted in an approximately 8-fold increase in MDM2 transcript levels and a 2-fold increase in the protein levels in lung. Cultured lung cells generated from these mice showed an approximately 7-fold increase in the MDM2 protein level after 24-hour Dox treatment (Figure 1D). Therefore, the levels of Dox-induced MDM2 expression in the lungs of tightMDM2 mice are comparable with reported MDM2 expression in human lung tumors (2). IHC analysis of formalin fixed paraffin embedded (FFPE) lung tissue sections from Dox-treated (+Dox) tightMDM2 mice confirmed Dox-induced MDM2 expression in the CCSP-expressing cells of respiratory bronchioles. Sucrose-treated (-Dox) mice or +Dox rtTA mice did not show significant MDM2 expression (Figure 1, B and E, and Supplemental Figures 1 and 2; supplemental material available online with this article; https://doi.org/10.1172/jci.insight.128194DS1). These data indicate that tightMDM2 transgenic mice express MDM2 in lung bronchioles after Dox treatment.

Since elevated MDM2 levels from CCSP promoter may induce proliferation of Club cells in lung tissue, expression of known proliferation markers by Dox-induced MDM2 in lung bronchioles was investigated by IHC staining of serial FFPE lung tissue sections from +Dox or -Dox tightMDM2 and +Dox rtTA mice. MDM2-expressing lung cells (Supplemental Figure 2A) around respiratory bronchioles showed more frequent and stronger expression of Ki67 (Supplemental Figure 2B) and phospho-histone H3 (p-H3; Ser10) (Supplemental Figure 2C) compared with that of -Dox tightMDM2 or +Dox rtTA mice, suggesting that Dox-induced MDM2 expression activated cycling of the CCSP-expressing bronchiolar cells. In addition, 


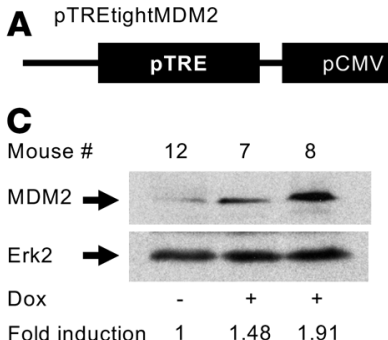

Fold induction $\quad 1 \quad 1.48 \quad 1.91$

\section{$\mathbf{E}$}

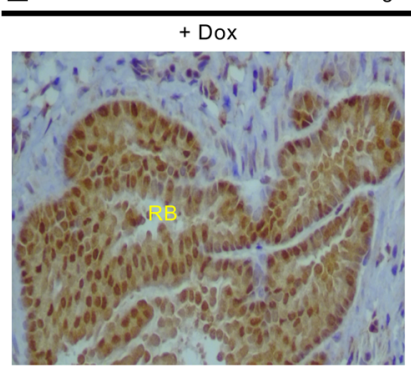

MDM2

D

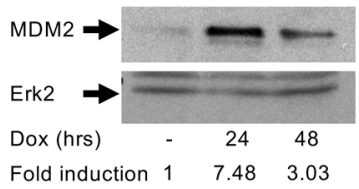

B

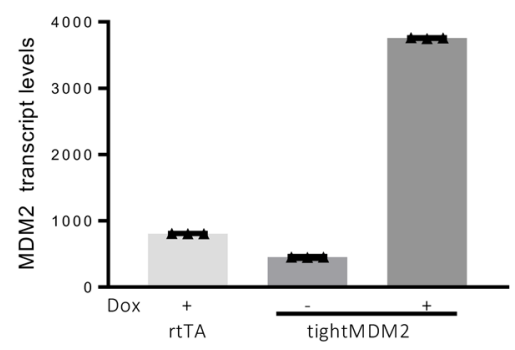

ghtMDM2
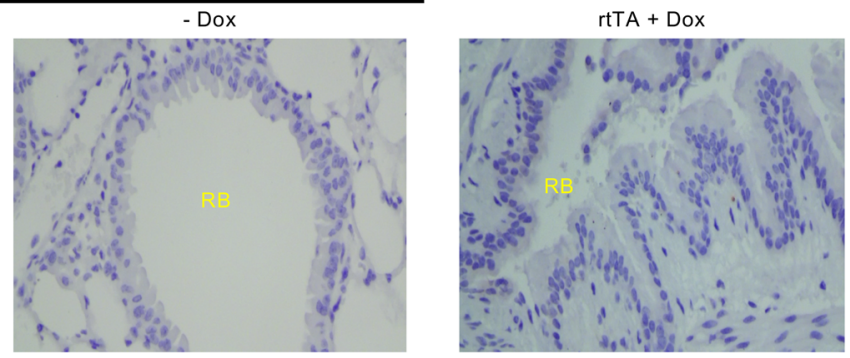

Figure 1. Generation of transgenic mice expressing human MDM2 in CCSP-expressing lung cells in response to Doxycycline (Dox). (A) pTREtightMDM2 construct designed to express MDM2 from a TRE-controlled CMV promoter. (B) MDM2 transcript levels normalized by GAPDH expression, representative data from 1 set of mice shown as mean \pm SD, and (C) MDM2 protein (immunoblot) expression in lung tissue from tightMDM2 (pTREtightMDM2:CCSPrtTA) or control rtTA (CCSPrtTA) transgenic mice after Dox or sucrose (-Dox) treatment. (D) MDM2 expression in cultured lung cells from tightMDM2 mice after 0,24 , or 48 hours of Dox treatment. Fold induction of MDM2 expression by Dox treatment in $\mathbf{C}$ and $\mathbf{D}$ were determined by densitometry and are shown at the bottom of the immunoblots. MDM2 transcript levels were determined by qPCR. MDM2 protein expression was determined by immunoblot analysis using an antibody against human MDM2. Erk2 was used as loading control. (E) Representative images (magnification, 20x) showing immunostained lung tissue sections from +Dox or -Dox tightMDM2 mice and +Dox rtTA mice using an antibody against human MDM2. MDM2 protein expression (brown) can be detected around the respiratory bronchiole (RB) after Dox treatment. All experiments were repeated in at least 3 mice.

lung tissue of +Dox tightMDM2 mice expressed higher levels of Cyclin D2 transcript compared with that of -Dox tightMDM2 or +Dox rtTA mice, indicating that Dox-induced MDM2 expression from CCSP promoter overrides the restriction point of the cell cycle (32) (Supplemental Figure 2D).

However, Ki67 expression can be observed in all active phases of the cell cycle except quiescent cells (33), and - in addition to chromosome condensation in mitotic cells - p-H3 has been implicated in phorbol ester-induced (12-O-Tertadeconoylphorbol-13-acetate-induced; TPA-induced) transcriptional activation in interphase cells (34). Therefore, the ability of Dox-induced MDM2 to activate DNA replication in CCSP-expressing cells was investigated. Introduction of a nucleotide analogue BrdU in +Dox or -Dox tightMDM2 mice and + Dox rtTA mice, however, did not show any BrdU incorporation in the respiratory bronchiole region of lung, despite efficient MDM2 expression (Supplemental Figure 2E). These data indicate that Dox-induced MDM2 expression from the CCSP promoter drives lung Club cells to overcome the restriction point of the cell cycle but not to $S$ phase DNA replication.

Elevated levels of MDM2 induce DNA replication consequent to depletion of Club cells after naphthalene inju$r y$. Depletion of Club cells is known to reactivate the regenerative potential of CCSP/SPC-dual-positive variant Club (vClub) and bronchioalveolar stem cells (BASC) lung progenitor cells (23-25). Therefore, we determined whether Dox-induced MDM2 expression from the CCSP promoter would augment proliferation of vClub and BASC cells during epithelial regeneration after naphthalene injury. Naphthalene treatment specifically depletes mouse Club cells due to cytotoxic compounds generated by an enzyme cytochrome P-450 2F2, but it spares naphthalene-resistant CCSP-expressing vClub or BASC, which lack the enzyme $(24,25)$.

Accordingly, +Dox tightMDM2 or rtTA and-Dox tightMDM2 mice were injected with naphthalene followed by BrdU and were harvested at 72 hours after naphthalene treatment and 5 hours after BrdU delivery. Consistent with the literature, IHC analysis of serial FFPE lung tissue sections showed the appearance of BrdU-incorporating cells in tightMDM2 or control groups after naphthalene treatment (Figure 2, A-C). 
However, +Dox tightMDM2 mice displayed doubled frequency of BrdU-incorporating cells with MDM2 and CCSP coexpression in comparison with the control groups (Figure 2, A-C). These data indicate that Dox-induced MDM2 expression activates DNA replication in naphthalene-resistant CCSP-expressing cells of lung bronchiole after naphthalene treatment. Analysis of p53 levels after naphthalene injury did not show appreciable alterations (Supplemental Figure 3, A and B). Thus, although MDM2 overexpression drives Club or alveolar epithelial cells to the restriction point of the cell cycle, it requires injury-induced signaling to motivate the cells to replicate DNA.

MDM2 induces expansion of CCSP-and SPC-labeled dual-positive progenitor cells in lung bronchiole and bronchioalveolar duct junction (BADJ), inducing rapid reepithelialization of lung bronchioles. Induction of DNA synthesis consequent to depletion of Club cells suggests proliferation of lung progenitor cells for injury repair $(23,35)$. Therefore, the consequence of Dox-induced MDM2 expression on expansion of lung progenitor cells (such as vClub and BASC cells), which restore bronchoalveolar epithelium, was examined. For this purpose, +Dox tightMDM2 or rtTA mice were treated with naphthalene. Since vClub and BASC cells express both Club cell marker CCSP and alveolar cell marker SPC, the presence of CCSP (cc10) and SPC coexpressing cells in the bronchiole and BADJ was investigated by immunostaining naphthalene-treated FFPE lung tissue sections with fluorescent dye-tagged antibodies. Consistent with the increase in DNA replicating cells, a robust expansion of CCSP/SPC-coexpressing cells in lung bronchioles and BADJ of +Dox tightMDM2 mice was observed compared with that of +Dox rtTA mice (Figure 2, D and E). Furthermore, the ability of Dox-induced MDM2 to accelerate restoration of the bronchiolar epithelium after naphthalene injury was examined by immunostaining and quantifying the fraction of bronchioles that restored CCSP-expressing Club cells. The results indicated that more than $69 \%$ of the bronchiole of + Dox tightMDM2 mice was consistently repopulated compared with that of +Dox rtTA mice, which showed $46 \%$ repopulation at 72 hours after naphthalene treatment (Figure 2, F and G). These data indicate accelerated restoration of bronchiolar epithelium by MDM2 after lung injury.

Increase in MDM2 expression is known to destabilize p53 (1), an event that may encourage S phase entry of CCSP-expressing lung cells. However, our data (Supplemental Figure 2E) show that increase in MDM2 expression did not initiate DNA replication in lung in the absence of naphthalene injury. We determined whether absence of $\mathrm{p} 53$ can mimic the effect of MDM2 expression in inducing DNA replication, proliferation of CCSP/SPC-dual-positive lung progenitor cells, and reepithelialization in lungs of p53-null mice after naphthalene injury. Immunostaining of FFPE lung tissue sections from naphthalene-treated WT p53 and p53-null mice, however, did not show any appreciable statistically significant acceleration of injury repair events in p53-null mice (Supplemental Figure 3, C-H). These results indicate that p53 depletion cannot serve as a surrogate for MDM2 in repairing lung injury.

MDM2 activates the signaling pathway for reepithelialization and injury repair. Naphthalene injury activates the cell signaling pathways for reepithelialization (36). Accelerated epithelial regeneration by Dox-induced MDM2 expression suggests that MDM2 may be a crucial factor for activation of injury-induced signaling pathways. Since MDM2 induced reepithelialization (Figure 2, F and G), and since it activates Akt phosphorylation and, thus, phosphorylation of GSK3 $\beta$ and Cyclin D2 expression in cultured lung cells $(37,38)$, the ability of MDM2 to activate Akt signaling during epithelial regeneration consequent to naphthalene injury was investigated. Dox-induced tightMDM2 and rtTA mice were treated with naphthalene. Immunoblot analysis of lung extracts from these mice revealed a robust increase in Akt phosphorylation at Ser 473 (Figure 3A and Supplemental Figure 4A) in extracts of tightMDM2 lungs compared with rtTA lungs, whereas immunostaining of the lung FFPE sections showed a drastic increase in phosphorylation of Akt substrates, GSK3 $\beta$ at Ser 9 (Figure 3, B and C), and $\beta$-catenin at Ser 552 (Figure 3, D and E), as well as nuclear translocation of phosphorylated $\beta$-catenin in $\mathrm{CCSP}^{+}$cells of lung bronchioles from +Dox tightMDM2 mice compared with that from + Dox rtTA mice. Unphosphorylated GSK3 $\beta$ or $\beta$-catenin did not show any significant change (Supplemental Figure 4, B and C). These observations indicate that Dox-induced MDM2 expression enhances Akt signaling consequent to naphthalene injury in $\mathrm{CCSP}^{+}$cells of lung bronchioles.

Inhibition of Akt abrogates MDM2-induced lung cell proliferation. To further explore the significance of MDM2-induced Akt phosphorylation on replication of bronchiolar progenitor cells, +Dox tightMDM2 mice were treated with an Akt inhibitor (MK-2206) after naphthalene injury. The effectiveness of the Akt inhibitor was ensured by examining the phosphorylation (at Ser9) of GSK3 $\beta$, a downstream target of Akt-mediated phosphorylation. The ability of the Akt inhibitor, MK226, to inhibit DNA replication was examined by analyzing BrdU incorporation in CCSP-expressing cells in lung bronchiole. Indeed, treatment with MK-2206 
A

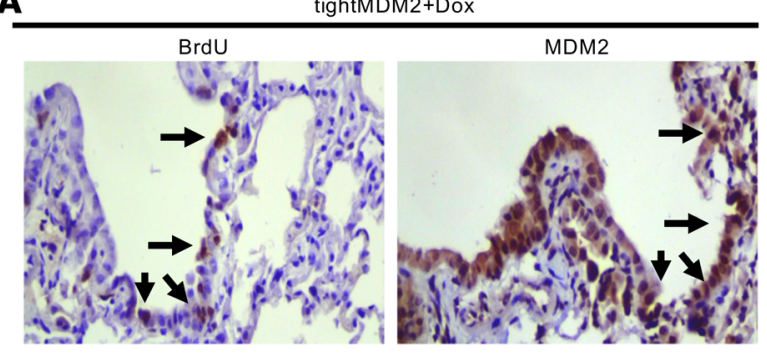

B

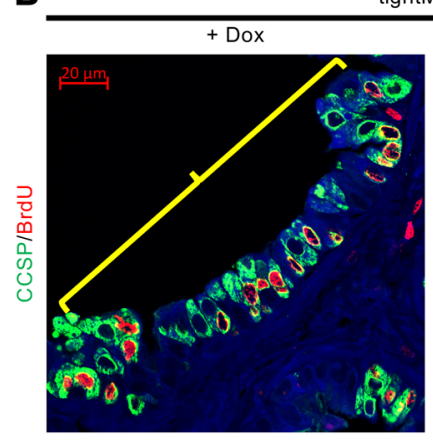

tightMDM2

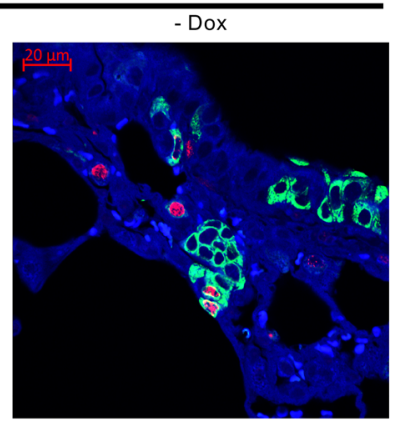

D
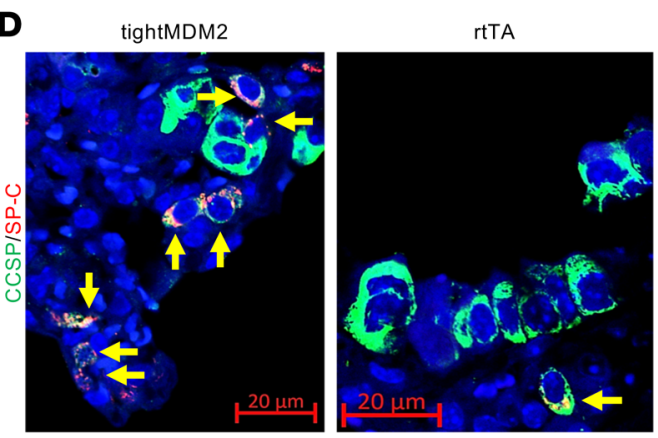

E

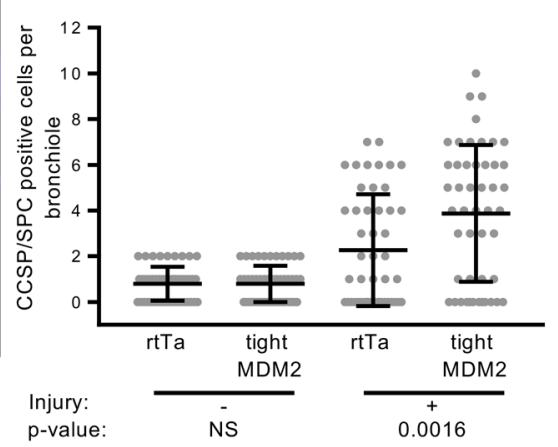

rtTA+Dox

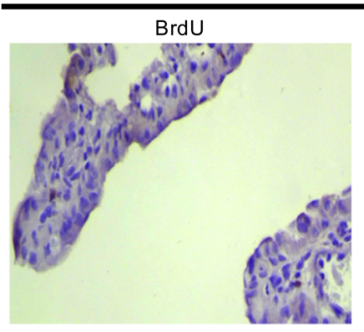

rtTA+ Dox

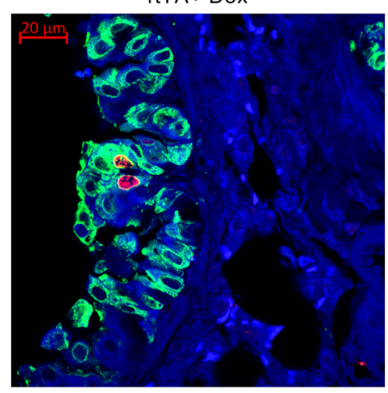

C

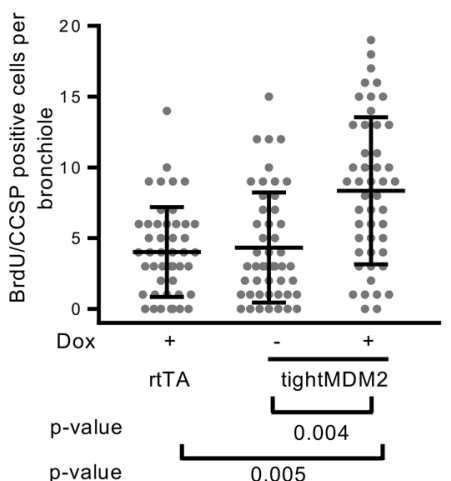

$\mathbf{F}$

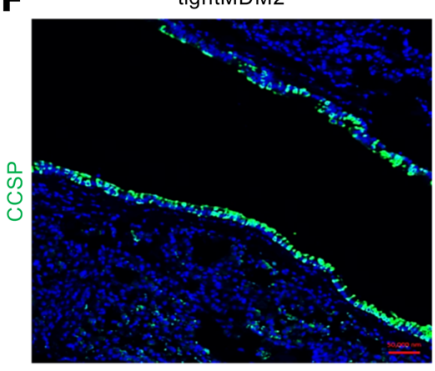

rtTA

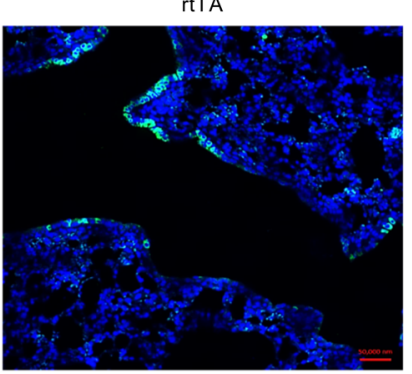

G

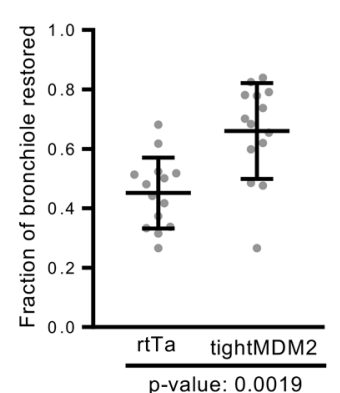

Figure 2. Dox-induced MDM2 expression in CCSP-expressing cells of mouse lung increases frequency of DNA-replicating lung progenitor cells, accelerating CCSP/SPC-dual-labeled progenitor cell proliferation and restoration of CCSP-expressing epithelial layer after naphthalene injury. (A) Representative images (magnification, 20x) show sequential FFPE lung tissue sections from Dox-treated tightMDM2 or rtTA mice after naphthalene-treatment and BrdU delivery immunostained using antibodies against human MDM2 and BrdU. Arrows indicate BrdU incorporation (brown) and MDM2 expression (brown) in similar region of lung bronchiole. (B) Representative images (magnification, 40x) show BrdU incorporation (red nuclear fluorescence) in CCSP-expressing (extranuclear green fluorescence) cells (bracket). (C) Frequency of BrdU incorporating CCSP+ cells in lung bronchioles of +Dox rtTA and -Dox or Dox+ tightMDM2 mice are shown by a dot plot. (D) Representative images (magnification, 40x) show CCSP/SPC-coexpressing (green and red, respectively) progenitor cells (arrows) in the lung bronchioles of Dox-treated tightMDM2 and rtTA mice detected by immunostaining. (E) Frequency of CCSP/SPC-coexpressing cells in the lung bronchioles are shown by a dot plot. Data for each treatment was collected from 3 mice, with 16 bronchioles per mice, and are plotted as mean \pm SD $(n=48)$. In all graphs, $P$ values calculated using Student's $t$ test are indicated. NS: not significant. (F) Representative images (magnification, $10 \times)$ show restoration of epithelial layers with CCSP-expressing cells in bronchioles of Dox-treated tightMDM2 and rtTA mice. (G) Dot plot comparing fraction of bronchioles restored in Dox-treated rtTA and tightMDM2 mice. Data for each treatment was collected from 3 mice, with 5 bronchioles per mice, and plotted as mean $\pm \mathrm{SD}(n=15)$. $P$ value calculated using Mann-Whitney-Wilcoxon test is indicated. 

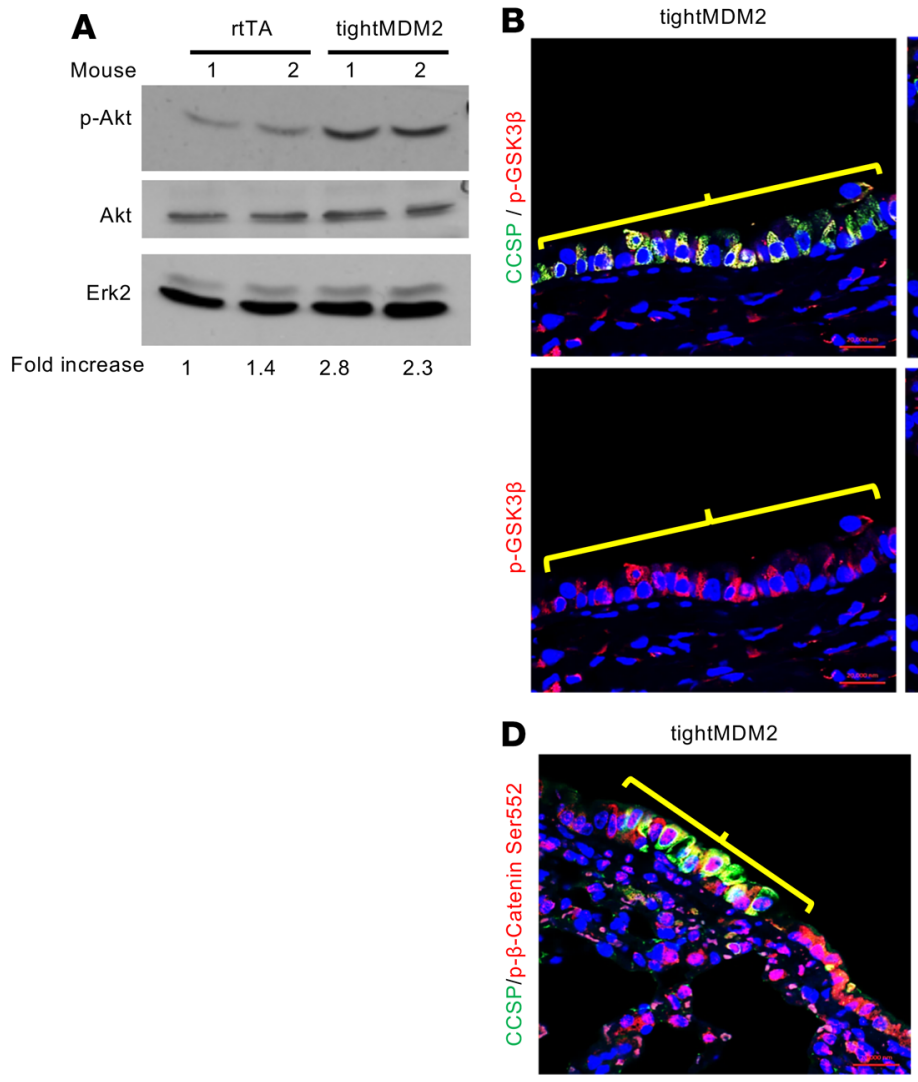
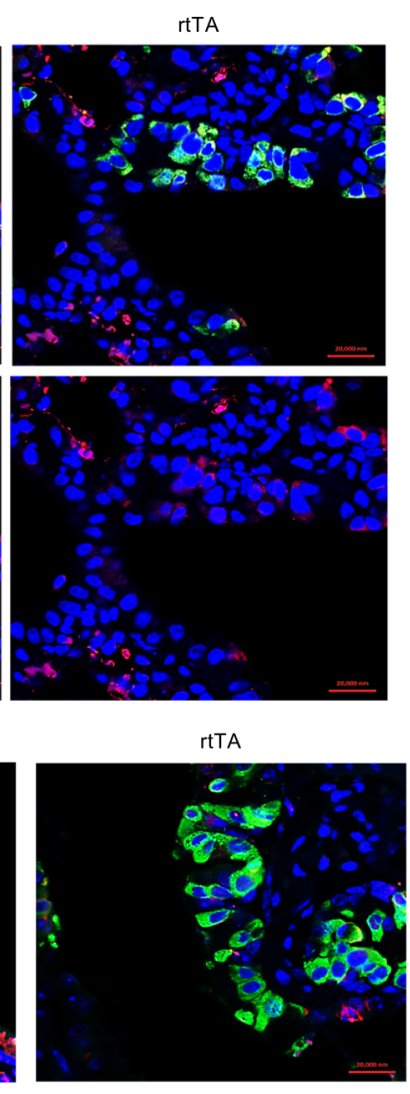
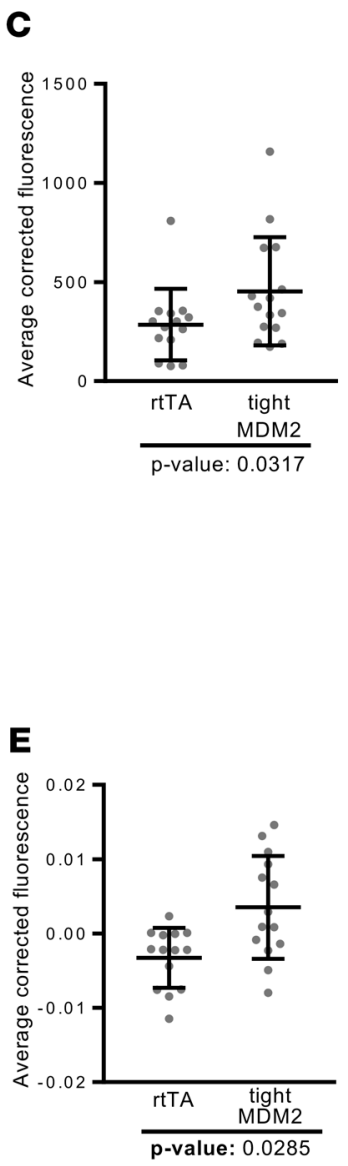

Figure 3. MDM2 activates the Akt signaling pathway consequent to naphthalene injury. Lung tissue extracts or FFPE tissue sections from Dox-treated rtTA and tightMDM2 mice were analyzed for activation of Akt signaling pathway 72 hours after naphthalene treatment. (A) Immunoblot analysis of Akt phosphorylation at Ser 473. Fold increase in p-Akt expression was determined by densitometry and is shown at the bottom of the immunoblots. (B) Representative images (magnification, 40x) show immunostained CSK3 $\beta$ phosphorylation at Ser 9 (red) in CCSP-expressing (green) cells. Positive cells are shown by brackets. (C) Dot plot comparing average fluorescence of each bronchioles. (D) Representative images (magnification, 40x) showing immunostained phospho (Ser 552) $\beta$-catenin (brackets) in CCSP-expressing cells. (E) Dot plot comparing average fluorescence of each bronchiole. Data for each treatment was collected from 3 mice, with 5 bronchioles per mice, and plotted as mean \pm SD $(n=15)$. In all graphs, $P$ values calculated using Student's $t$ test are indicated.

reduced downstream GSK3 $\beta$ phosphorylation (Figure 4A), as expected. Furthermore, MK-2206 also reduced the number of BrdU-labeled CCSP ${ }^{+}$cells in bronchioles of +Dox tightMDM2 mice (Figure 4, B and C), indicating that Akt signaling is needed for MDM2-induced proliferation of CCSP-expressing cells. Unlike the reported upregulation $(39,40)$ or downregulation $(41)$ of WT p53 by PI3K inhibitor in cultured cells, inhibition of Akt by MK-2206 did not significantly alter p53 levels in lung bronchiole (Supplemental Figure 5).

We have reported earlier (37) that, in cultured cells, elevated MDM2 levels hasten S phase entry of cells in the absence of p53 using a PI3-kinase-dependent pathway. Since cultured cells are exposed to growth media supplemented with growth factors, we determined whether the Akt signaling pathway is required for MDM2-induced DNA replication and wound healing. For this purpose, we introduced siRNA against Akt (Akt siRNA) into early passage lung cells isolated from p53-null (p53--) and p53-null:MDM2 transgenic (p53--:MDM2Tg) mice (37). The effect of Akt depletion on wound healing and DNA replication efficiency of these cells were examined using wound healing assay and BrdU incorporation analysis. Our data

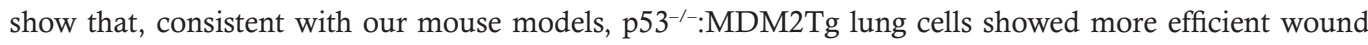
healing and DNA replication abilities than $\mathrm{p} 53^{-/-}$lung cells. Introduction of Akt siRNA inhibited these functions (Supplemental Figure 6). These data indicate that MDM2-induced wound healing and cell proliferation require the Akt signaling pathway. Ability of MDM2 to activate Akt signaling and the involvement of this property for efficient lung progenitor cell or cultured lung cell proliferation (Figure 3 and Figure 4 and ref. 38) signify that inhibition of Akt signaling in the context of MDM2 overexpression may prevent deregulated progenitor cell proliferation during repair of lung injury. 
A
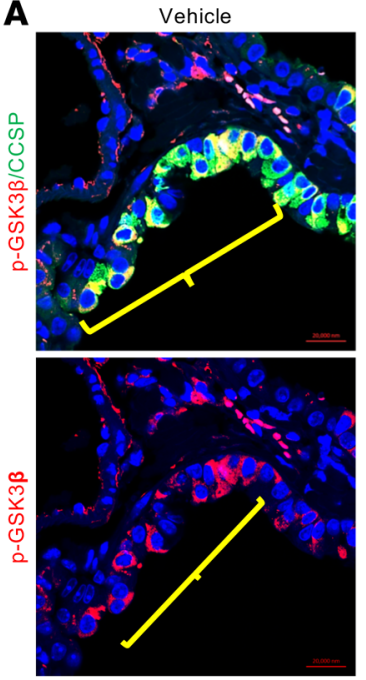

MK-2206
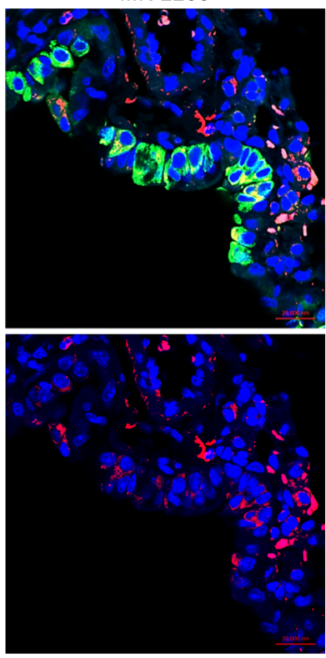

B

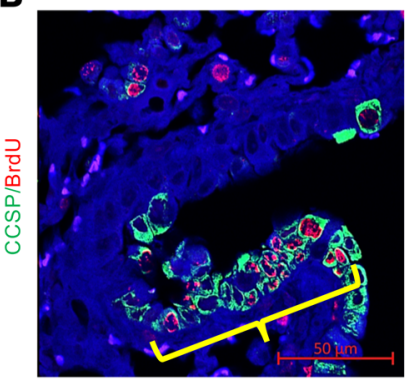

C

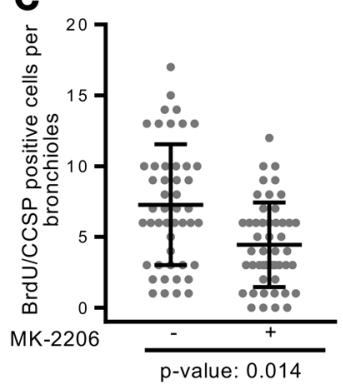

MK-2206

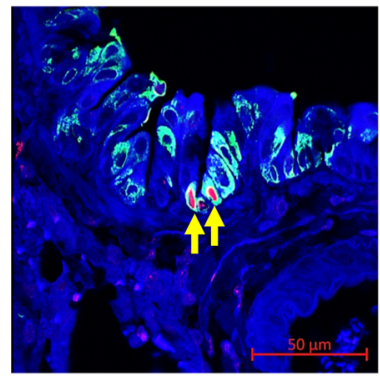

Figure 4. Inhibition of Akt abrogates MDM2-induced proliferation of CCSP-expressing lung cell after naphthalene injury. (A) Representative images (magnification, 40x) show immunostained CSK3 $\beta$ phosphorylation at Ser 9 (red) in CCSP expressing (green) cells. (B) Representative images (magnification, 40x) showing BrdU incorporation (red) in CCSP expressing (green) cells. Positive cells are shown by brackets. (C) Frequency of BrdU incorporating (red) $\mathrm{CCSP}^{+}$(green) cells in the absence or presence of MK-2206 were counted and are shown by a dot plot. $P$ value calculated using Student's $t$ test is indicated. Data for each treatment were collected from $3+$ Dox tightMDM2 mice, with 16 bronchioles per mice, and plotted as mean \pm SD $(n=48)$

MDM2 activates the NOTCH signaling pathway consequent to naphthalene injury. Since Notch-1 is required for regeneration of Club cells during airway injury repair (42), and GSK3 $\beta$ regulates Notch-1 function (43), ability of MDM2 to induce Notch-1 expression and nuclear translocation of Notch intracellular domain (NICD) in Club cells during injury repair was investigated. Accordingly, FFPE lung sections from Dox-treated, tightMDM2 and rtTA mice were examined. In addition, expression of MDM2, Notch-1, and the downstream transcriptional target of activated Notch-1, HES-1, transcript levels in the lungs of these mice were also analyzed. Indeed, Dox-induced MDM2 expression promoted expression of Notch-1 protein and transcripts, and nuclear translocation of NICD in $\mathrm{CCSP}^{+}$cells (Figure 5, A-C) activating expression of HES-1 (Figure 5D). These data indicate that MDM2 activates Notch-1 signaling pathways after naphthalene injury in lung.

MDM2 enhances expression of EMT markers consequent to naphthalene-induced lung injury. Since epithelial regeneration after naphthalene-induced lung injury should involve EMT, expression of EMT markers such as vimentin, $\mathrm{N}$-cadherin, and E-cadherin - in $\mathrm{CCSP}^{+}$lung progenitor cells was investigated in FFPE lung sections of Dox-treated tightMDM2 and rtTA mice. The results indicated a remarkable increase in $\mathrm{N}$-cadherin (Figure 6A) and vimentin (Figure 6, B and C) and decrease in E-cadherin (Supplemental Figure 7) expression in CCSP-expressing cells in the bronchiole of Dox-treated tightMDM2 mice compared with rtTA control. These data indicate that induction of MDM2 expression in CCSP-expressing lung cells leads to EMT after naphthalene injury, generating cells positive for mesenchymal/fibroblast markers.

MDM2-induced progenitor cell proliferation and reepithelialization of lung bronchioles after naphthalene injury takes place in gain-of-function p53 mutant R172H knock-in mice. Increased frequency of proliferating lung progenitor cells by MDM2 could be a result of its ability to inactivate WT p53 (1). Since lung cancers with p53 mutation often show MDM2 overexpression $(2,21,44)$, the ability of MDM2 to induce DNA replication, expansion of CCSP/SPC-dual-positive progenitor cells, and reepithelialization was determined in the presence of mutant p53. tightMDM2, lox-stop lox (LSL) R172H (knock-in) mice were generated. IHC analysis of serial FFPE lung sections of these Dox-treated tightMDM2, LSL R172H and control LSL R172H mice showed coexpression of p53 mutant R172H and MDM2 in lung bronchioles of tightMDM2, LSL R172H mice after Dox induction (Figure 7A). Consistent with tightMDM2 mice lungs, bronchioles of +Dox tightMDM2, LSL R172H mice showed higher frequency of interphase cells expressing Ki67 and 
A
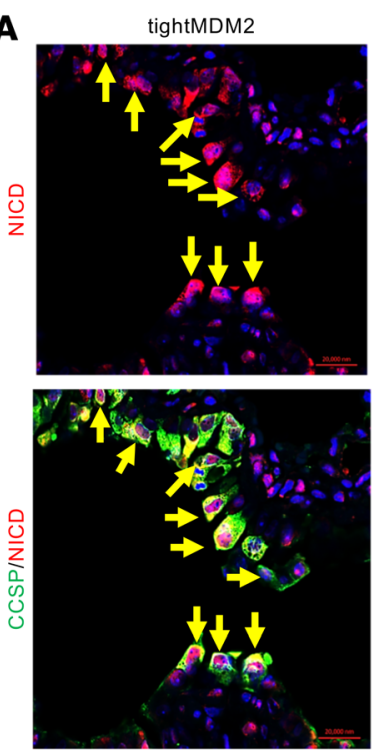

C

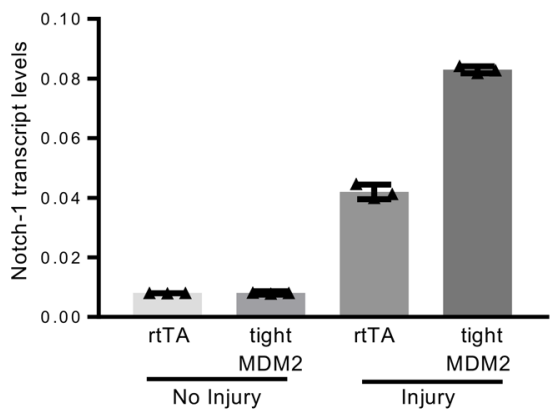

rtTA
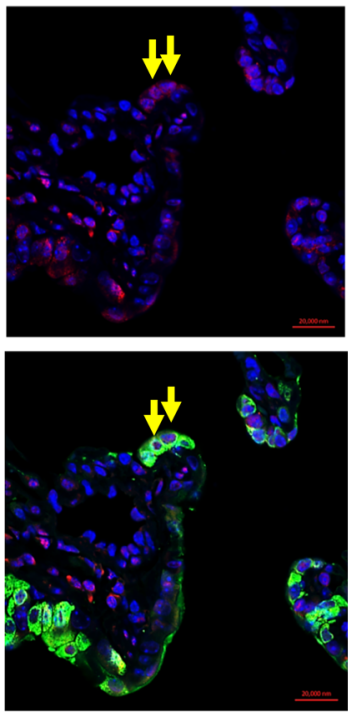

D

\section{B}

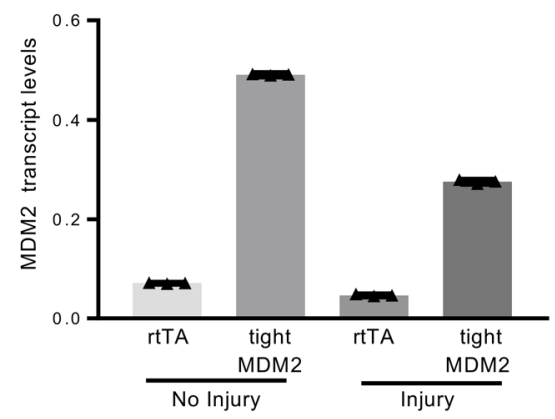

Figure 5. MDM2 activates the NOTCH signaling pathway consequent to naphthalene injury. Lung tissue extracts or FFPE tissue sections from Dox-treated rtTA and tightMDM2 mice were analyzed for NOTCH-1 expression, nuclear translocation of Notch intracellular domain (NICD), and HES-1 expression in CCSP expressing lung bronchiole 72 hours after naphthalene treatment. (A) Representative images (magnification, 40x) showing activated Notch-1-, NICD- (red nuclear fluorescence), and CCSP+ (green extranuclear fluorescence) cells detected by immunostaining (double-positive cells shown by arrows). (B) MDM2, (C) Notch-1, and (D) HES-1 transcript levels in lung tissue determined by qPCR. Representative data from one set of mice plotted as mean \pm SD. All experiments were repeated in at least 3 mice.

p-H3 markers (Supplemental Figure 8A) compared with +Dox LSL R172H controls, but BrdU delivery did not show BrdU-incorporating cells (Supplemental Figure 8B). Naphthalene-mediated Club cell depletion initiated BrdU incorporation in CCSP-expressing lung cells of LSL R172H control or tightMDM2, LSL R172H mice, while bronchioles from naphthalene-treated +Dox tightMDM2, LSL R172H mice coexpressing MDM2 and $\mathrm{R} 172 \mathrm{H}$ displayed a robust increase in the frequency of BrdU-labeled CCSP ${ }^{+}$cells (Figure 7, B and C) and activated expansion of CCSP/SPC-dual-positive cells (Figure 7, D and E) compared with bronchioles from LSL R172H control mice expressing R172H alone. Furthermore, CCSP staining of naphthalene-treated lung bronchioles of +Dox tightMDM2, LSL R172H mice showed a robust increase in the efficiency of reepithelialization compared with LSL R172H bronchioles (Figure 7, F and G). These data indicate that MDM2 activates epithelial repair in the presence of gain-of-function p53 mutant $\mathrm{R} 172 \mathrm{H}$ in the absence of WT p53 after naphthalene-induced depletion of Club cells.

Targeted induction of MDM2 expression in lung alveoli activates DNA replication after treatment with ionizing radiation. To determine whether induction of MDM2 expression in lung alveolar type II (ATII) cells activates DNA replication after injury, transgenic pTREtightMDM2 SPC-rtTA (SPC-tightMDM2) mice were generated by crossbreeding of pTREtightMDM2 with transgenic SPC-rtTA mice. SPC-tightMDM2 mice express rtTA in lung ATII cells and, thus, induce MDM2 expression in SPC-expressing cells after treatment with Dox (Supplemental Figure 9A). Consistent with the results found for lung bronchiole, FFPE 
A
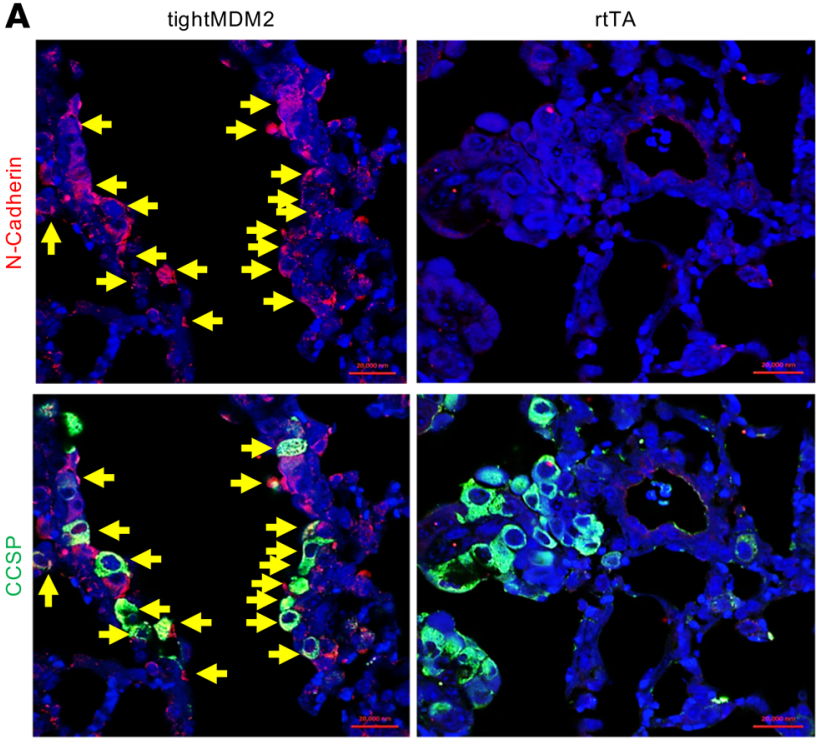

B
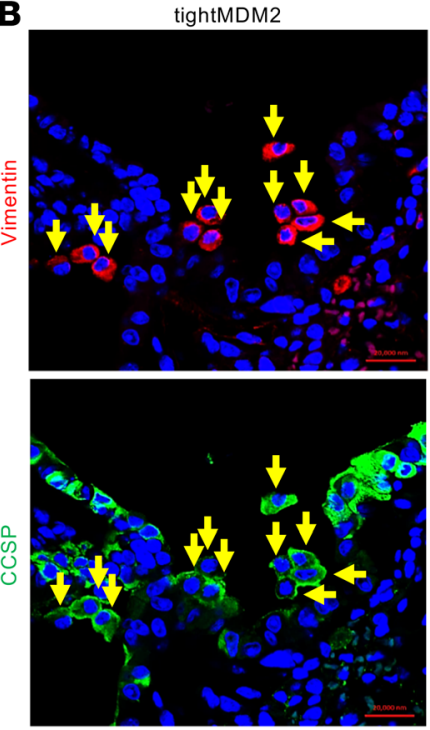
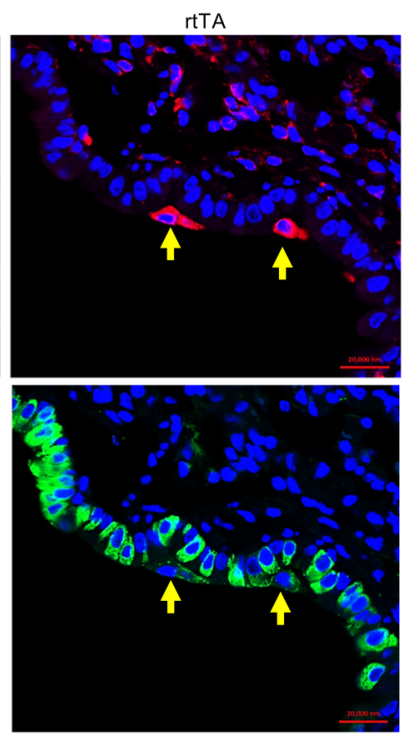

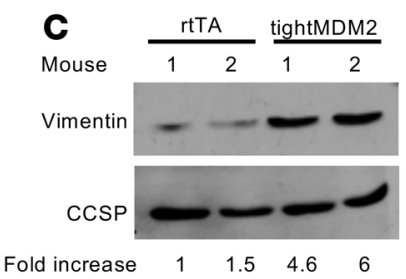

Figure 6. MDM2 enhances expression of EMT markers, $\mathbf{N}$-cadherin, and vimentin, consequent to naphthalene-induced lung injury. (A-C) Representative images (magnification, 40x) of FFPE lung sections after immunostaining of (A) N-cadherin (arrows) and (B) vimentin (arrows) in CCSP expressing (green) cells of lung bronchiole, and (C) representative immunoblot analysis of vimentin in lung tissue extracts. Fold increase in vimentin expression was determined by densitometry and is shown at the bottom of the immunoblot. All experiments were repeated in at least 3 mice.

lung sections from +Dox SPC-tightMDM2 mice showed increased Ki67 expression in alveolar epithelial cells compared with -Dox SPC-tightMDM2 or +Dox SPC-rtTA mice (Supplemental Figure 9B). Also, no BrdU incorporation was observed in any of the constructs after BrdU delivery (Supplemental Figure 9B). To determine the contribution of MDM2 in ATII cell proliferation in response to lung injury, SPC-tightMDM2 and control SPC-rtTA mice were exposed to ionizing radiation (14 Gy). Since BrdU labeling in ATII cells of a mouse lung can be observed at 3 months after irradiation (45), contribution of MDM2 in ATII cell proliferation was determined using BrdU incorporation at 3 months after irradiation. Consistent with the results observed for naphthalene-induced Club cell depletion, appearance of BrdU+ SPC-expressing cells was observed 3 months after radiation exposure in the lung alveolar region of all irradiated mice. However, +Dox SPC-tightMDM2 lung FFPE sections indeed showed an increase in the frequency of BrdU-labeled SPC ${ }^{+}$alveolar cells compared with that from +Dox SPC-rtTA or -Dox SPC-tightMDM2 mice (Figure 8, A and B). Furthermore, +Dox SPC-tightMDM2 lung sections also showed an increase in vimentin-expressing ATII cells compared with ATII cells of irradiated control groups (Figure 8, C and D). As reported in the literature $(46,47)$, induction of p53 was not observed in lung sections harvested 3 months after radiation (Supplemental Figure 9C). These results indicate that MDM2 induces proliferation and EMT of ATII cells after radiation injury.

Endogenous MDM2 is essential for progenitor cell proliferation and epithelial regeneration after lung injury. The ability of Dox-induced MDM2 to activate repair of naphthalene-induced lung injury raised the possibility that MDM2 is needed for progenitor cell proliferation and epithelial repair after lung injury. Since loss of both alleles of mdm2 is embryonically lethal in the presence of WT p53, efficiency of epithelial repair after naphthalene-induced depletion of Club cells were investigated in $\mathrm{p} 53^{-/-}: \mathrm{Mdm}^{-/-}$mice (Supplemental Figure 10, A-D). Results of our repeated experiments revealed that $\mathrm{p} 53^{-/-}: \mathrm{Mdm}^{-/-}$mice were extremely vulnerable to naphthalene treatment and, unlike $\mathrm{p} 53^{-/-}: \mathrm{Mdm} 2^{+/+}$mice, did not survive beyond 48 hours. Consistent with the results (Supplemental Figure 2) from lungs of Dox-inducible rtTA 
A

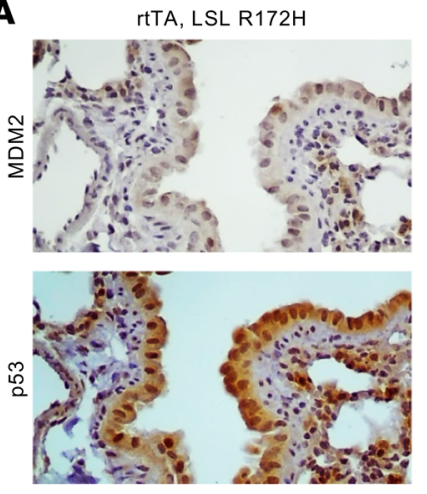

C

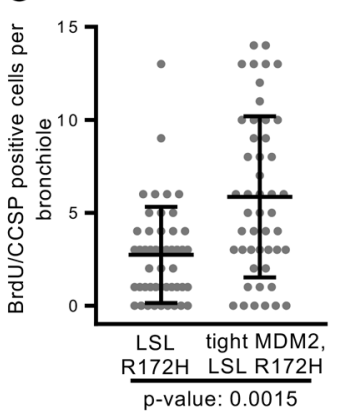

F

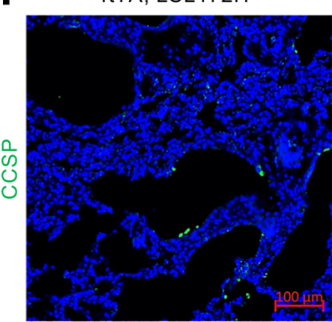

D

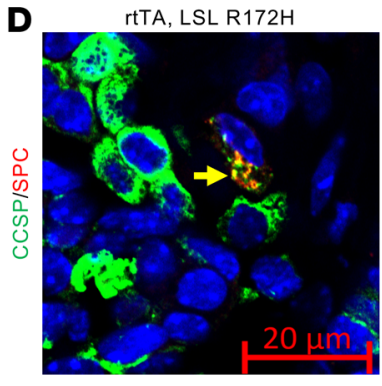

B

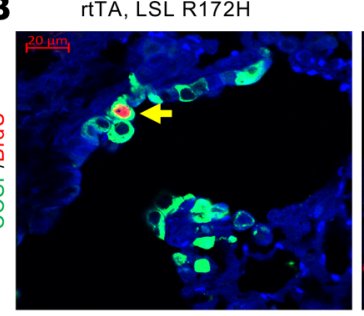

tightMDM2, LSL R172H

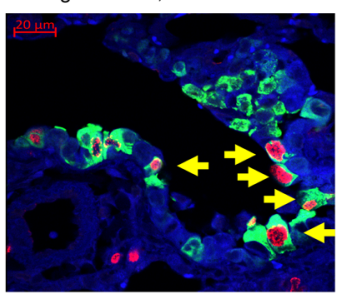

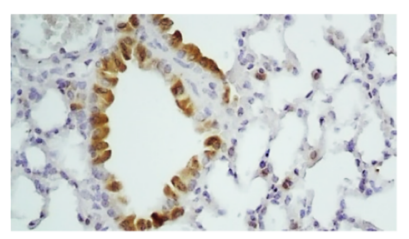

tightMDM2, LSL R172H
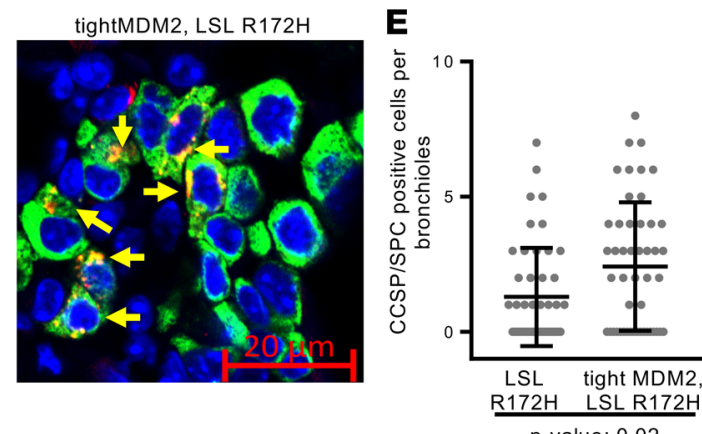

p-value: 0.02

tightMDM2, LSL172H

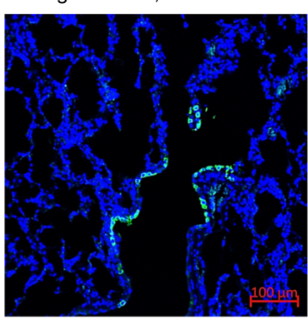

G

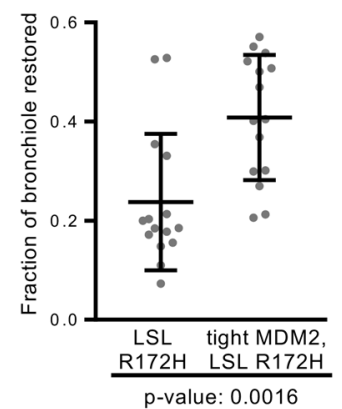

Figure 7. MDM2-induces DNA replication, progenitor cell proliferation, and reepithelialization in lung bronchioles of R172H knock-in mice after naphthalene injury. FFPE lung sections from Dox-treated LSL R172H or LSL R172H tightMDM2 mice were analyzed by immunostaining after naphthalene injury and BrdU delivery. (A) Representative images (magnification, 20x) showing Dox-induced coexpression (brown) of MDM2 and mutant p53-R172H in bronchioles detected by immunostaining of serial lung tissue sections. (B) BrdU incorporation (red nuclear) in CCSP+ (green extranuclear) cells in lung bronchiole detected by immunostaining (magnification, 40x). (C) Frequency of BrdU-incorporating CCSP+cells are shown by a dot plot. (D) Representative images (magnification, 40x) showing CCSP/SPC-coexpressing (green and red, respectively) progenitor cells (arrows) in lung bronchioles. (E) Frequency of CCSP/SPC-coexpressing cells in lung bronchiole are compared by a dot plot. Data for each treatment (C-E) was collected from 3 mice, with 16 bronchioles per mice, and plotted as mean \pm SD ( $n=48)$. In all graphs, $P$ values calculated using Student's $t$ test are indicated. (F) Representative images (magnification, 10x) showing restoration of epithelial layer with CCSP+ cells in lung bronchioles 72 hours after naphthalene injury. (C) Fraction of bronchioles restored has been compared using dot plot. Data for each treatment was collected from 3 mice, with 5 bronchioles per mice, and plotted as mean \pm SD ( $n=15)$. $P$ value calculated using Mann-Whitney-Wilcoxon test is indicated.

and tightMDM2 mice, lungs from $\mathrm{p} 53^{-/-}: \mathrm{Mdm}^{-/-}$mice showed fewer $\mathrm{Ki}^{+} 7^{+}$and $\mathrm{p}-\mathrm{H} 3^{+}$bronchiolar cells and reduced expression of Cyclin D2 transcripts compared with lungs from $\mathrm{p} 53^{-/-}: \mathrm{Mdm}^{+/+}$mice (Supplemental Figure 11, A and B). BrdU incorporation analysis at 48 hours after naphthalene treatment showed the appearance of BrdU-labeled CCSP-expressing cells only in the bronchioles of $\mathrm{p} 53^{-/-}: \mathrm{Mdm} 2^{+/+}$mice, but not in $\mathrm{p} 53^{-/}: \mathrm{Mdm} 2^{-/-}$mice (Figure 9, $\mathrm{A}$ and $\mathrm{B}$ ), indicating that the presence of MDM2, but not p53, is needed for initiating DNA replication in lung progenitor cells after Club cell depletion. Consistent with the results from lungs of naphthalene-treated rtTA and tightMDM2 mice, lungs from naphthalene-treated p53-/-:Mdm2 $2^{-/-}$mice showed $\mathrm{CCSP}^{+}$cells displaying reduced frequency of phosphorylation of GSK3 $\beta$, a target of Akt; NICD nuclear translocation; and reduced expression of Notch-1 and HES-1 transcripts 

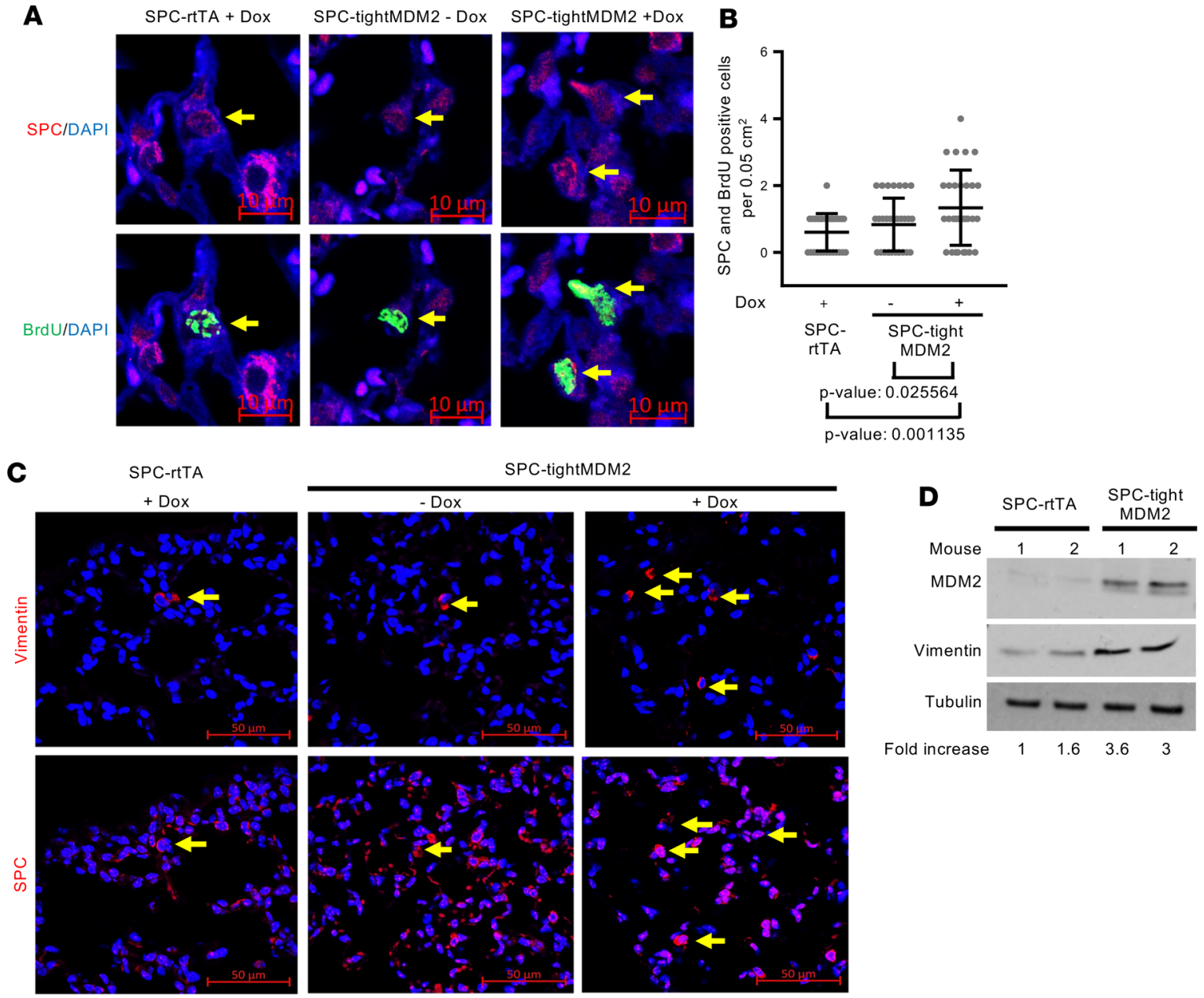

Figure 8. Induction of MDM2 expression in lung alveolar cells from a Dox-inducible SPC promoter increases frequency of DNA-replicating alveolar progenitor cells in SPC-tightMDM2 mice after exposure to ionizing radiation. FFPE lung tissue sections or lung tissue extracts from +Dox SPC-rtTA and -Dox or +Dox SPC-tightMDM2 mice harvested 3 months after exposure to ionizing radiation and BrdU delivery were analyzed. (A) Representative images (magnification, 40x) of immunostained sections show BrdU-incorporating (green nuclear) SPC-expressing (red extranuclear) cells (arrows). (B) Frequency of $\mathrm{BrdU}^{+}$SPC-expressing cells is shown by a dot plot. $P$ values calculated using Student's $t$ test are indicated. Data for each treatment was collected from 3 mice, with 10 blind alveolar regions per mice, and plotted as mean \pm SD $(n=30)$. (C) Representative images (magnification, 40x) of immunostained serial lung tissue sections show vimentin expression (red) in SPC-expressing cells (red). Arrows indicate similar areas in the serial sections. (D) Representative immunoblot analysis to quantify vimentin in lung tissue extracts. Fold increase in vimentin expression was determined by densitometry and is shown at the bottom of the immunoblot. Experiment was performed in 3 sets of mice.

compared with the lungs of $\mathrm{p} 53^{-/-}: \mathrm{Mdm} 2^{+/+}$mice (Supplemental Figure 11, C-F). Consistent with literature (48), immunostaining of FFPE lung sections revealed that 48 hours after naphthalene injury was not adequate for visualizing expansion of CCSP/SPC double-positive cells in lung bronchiole.

Since our data show that the ability of MDM2 to initiate DNA replication in lung progenitor cells after naphthalene-induced Club cell depletion does not require WT p53 (Figure 7 and Figure 9, A and B), we examined the efficiency of epithelial repair after naphthalene injury in $\mathrm{p} 53^{+/-}: \mathrm{Mdm} 2^{+/-}$mouse lungs compared with $\mathrm{p}^{+/--}: \mathrm{Mdm} 2^{+/+}$controls (Supplemental Figure 10) 72 hours after naphthalene treatment, followed by BrdU delivery. IHC analysis of FFPE lung tissue sections from naphthalene-treated $\mathrm{p} 53^{+/}$ $: M d m 2^{+/-}$mice showed a sharp reduction of BrdU-labeled $\mathrm{CCSP}^{+}$cells (Figure 9, C and D) and a consistent decrease in CCSP/SPC-dual-positive cells in the bronchiole and BADJ compared with sections from p53 $3^{+/-}: \mathrm{Mdm}^{+/+}$mice (Figure 9, E and F). In addition, the fraction of bronchioles that restored CCSP-expressing Club cells was less in $\mathrm{p} 53^{+/-}: \mathrm{Mdm}^{+/-}$mice compared with that from $\mathrm{p} 53^{+/-}: \mathrm{Mdm} 2^{+/+}$control mice, indicating reduced efficiency of reepithelialization (Figure 9, G and $\mathrm{H}$ ). These data demonstrate that loss of 1 endogenous $M d m 2$ allele impedes repopulation of bronchiolar epithelium after naphthalene injury, signifying a crucial role of MDM2 in lung injury repair. Taken together, the data presented in Fig- 
A
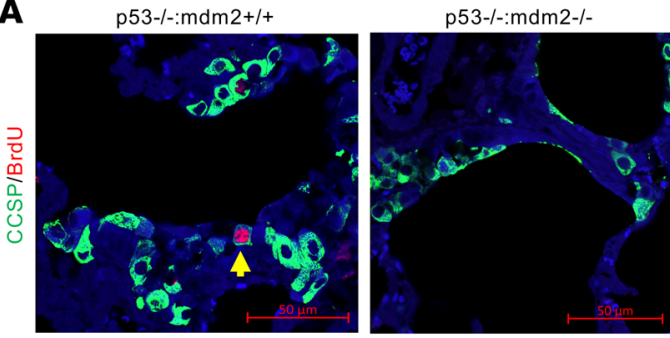

C
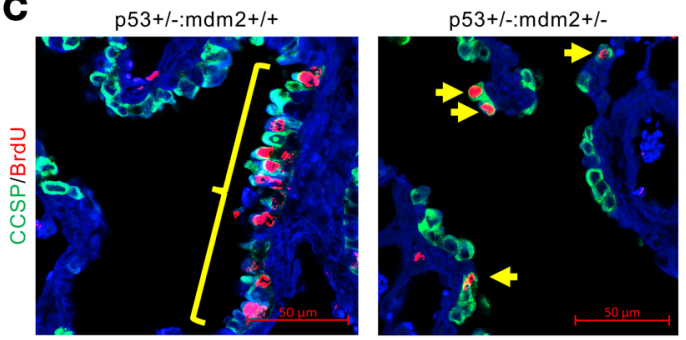

$\mathbf{E}$
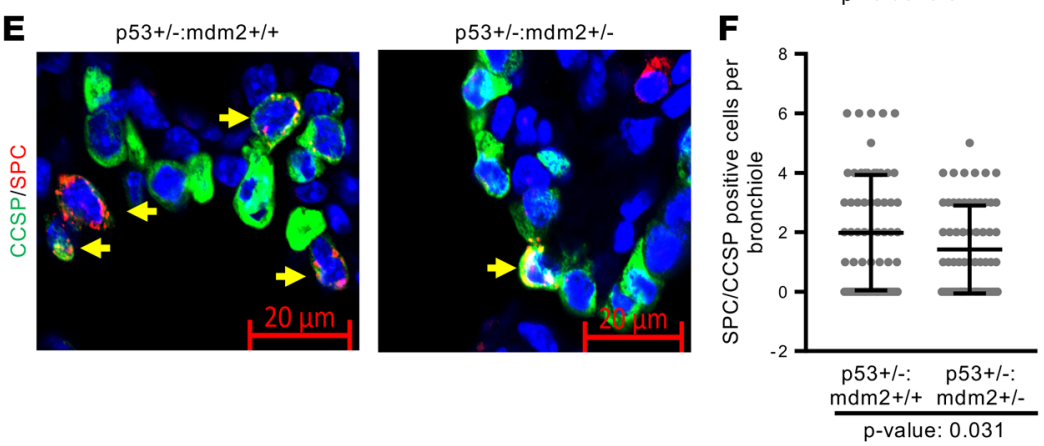

$\mathbf{G}$

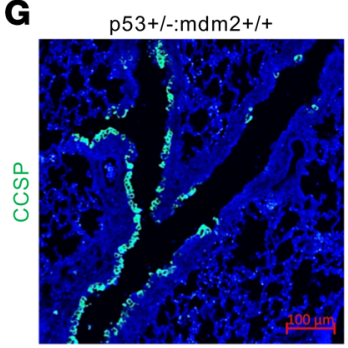

B

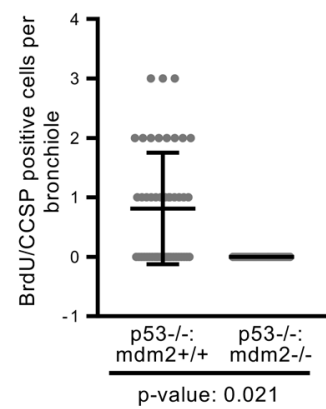

D

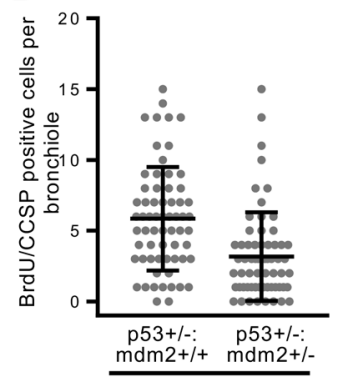

p-value: 0.022

H

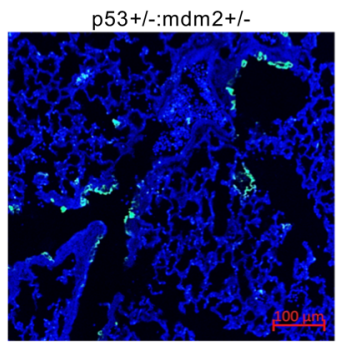

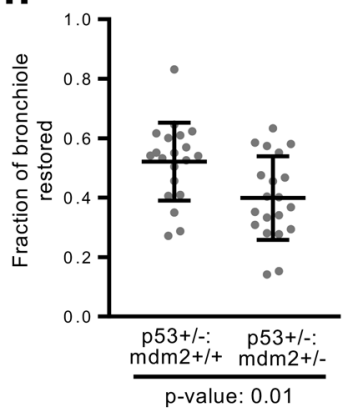

Figure 9. MDM2 is essential for DNA replication and proliferation of lung progenitor cells after naphthalene injury. FFPE lung sections of indicated mouse constructs were analyzed by immunostaining after naphthalene injury and BrdU delivery. ( $A$ and $\mathbf{C}$ ) Representative images (magnification, 40x) showing BrdU incorporation (red) in CCSP-expressing (green) cells in lung bronchioles. (B and $\mathbf{D})$ Dot plot showing frequency of BrdU-incorporating CCSP+ cells. Since

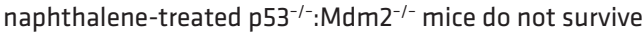
for 72 hours, data for $\mathbf{A}$ and $\mathbf{B}$ was collected at 48 hours of naphthalene treatment. Thirty-eight BrdU/CCSP-

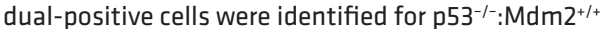
and none for $\mathrm{p}^{-3^{--}}: \mathrm{Mdm2}^{-/-}$mice in 48 bronchioles from 3 mice. (E) Representative images (magnification, 40x) showing CCSP/SPC-coexpressing (green and red, respectively) progenitor cells (arrows) in lung bronchioles. (F) A dot plot showing frequency of CCSP/SPC-coexpressing cells in lung bronchioles. Data for each treatment were collected from 3 mice, with 16 bronchioles from each mouse, and plotted as mean \pm SD $(n=48)$. In all graphs, $P$ values calculated using Student's $t$ test are indicated. (C) Representative images (magnification, 10x) showing restoration of epithelial layer with CCSP-expressing cells in lung bronchioles. (H) Dot plot to demonstrate fraction of bronchioles restored. Data for each treatment was collected from 3 mice, with 5 bronchioles per mouse, and plotted as mean \pm SD $(n=15)$. $P$ value calculated using Mann-Whitney-Wilcoxon test is indicated.

ure 9 show that MDM2 is not only a crucial factor for progenitor cell proliferation, but also enhances this function when overexpressed.

\section{Discussion}

Although the human oncoprotein MDM2 is frequently overexpressed in human lung cancer, there is no animal model to demonstrate its cell proliferative activity in lung. Results presented in this study demonstrate that MDM2 is required for DNA replication and proliferation of lung progenitor cells during epithelial regeneration after lung injury. We present data that demonstrate the ability of MDM2 to induce proliferation of lung progenitor cells by generating Dox-inducible mouse models that allow controlled elevation of MDM2 expression in either CCSP-expressing cells, which includes CCSP/SPC-dual-positive cells in lung bronchiole, or SPC-expressing alveolar epithelial cells, which include ATII cells (Figures 2 and 8 and 
Supplemental Figure 9). Increased MDM2 expression led to initiation of DNA replication only in lung progenitor cells (Figure 2B, Figure 7B, and Figure 8A) and only in response to naphthalene or radiation injury (Figure 2, Figure 7B, Figure 8, Supplemental Figure 2E, Supplemental Figure 8, and Supplemental Figure 9). The DNA replication event was followed by proliferation, expansion and EMT of lung progenitor cells, and rapid repopulation of lost epithelial layers (Figures 2 and 6-8). Our investigation to determine the role

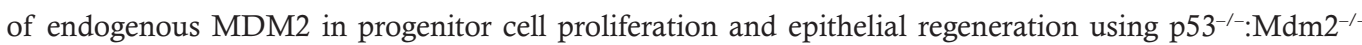
mice revealed that MDM2 is an essential component of progenitor cell proliferation for epithelial repair after lung injury. In addition, we present data to show that loss of $1 \mathrm{Mdm} 2$ allele in $\mathrm{p}^{53^{+/-}}$mice impedes proliferative activities of lung progenitor cells and restoration of bronchiolar epithelium, implicating that MDM2 is a crucial component needed for epithelial restoration after lung injury (Figure 9).

However, MDM2 alone was not sufficient to induce progenitor cell proliferation, which requires an injury-induced signaling pathway, as reported in the literature $(36,49)$. The presented data indicate that, although MDM2 overexpression upregulates Cyclin D2 expression and drives Club or alveolar epithelial cells to the restriction point of the cell cycle (Supplemental Figure 2, Supplemental Figure 8, Supplemental Figure 9B, and Supplemental Figure 11, A and B), it requires injury-induced signaling to motivate the cells to replicate DNA. Proliferative activity in adult lungs are mostly detected as a response to injury (24), which is known to induce secretion of growth factors, eliciting the injury signaling pathway, and causing progenitor cell proliferation and reepithelialization $(36,49)$. Our data show that MDM2 is not only essential for this basal proliferative activity (Figure 9), but it also enhances this function when overexpressed (Figure 10). Increased or sustained exposure to growth factors could complement cell proliferative activities of MDM2. Arguing for this hypothesis, cooccurrence of MDM2 and growth factor overexpression in human cancers (50-52) has often been reported, signifying their mutual cooperation during tumorigenesis. Furthermore, our observations are consistent with earlier reports suggesting resistance of cancer cells with MDM2 overexpression to radiation therapy $(1,4)$.

We reported earlier that MDM2 induces Akt phosphorylation independently of p53 (38). Our data presented in this communication indicate that this activity of MDM2 is needed for inducing DNA replication in lung progenitor cells and reepithelialization of bronchiolar epithelium. Dox-induced expression of MDM2 activated repair of lung injury, while inhibition of MDM2-induced Akt signaling prevented proliferation of lung progenitor cells that endogenously overexpresses MDM2 (Figure 3 and Figure 4). These findings are consistent with the reports that Akt activates cyclin-dependent kinases (downstream of p53-induced growth arrest) by phosphorylation of p21 preventing CDK2 or CDK4 inhibition (53). However, the Akt inhibitor did not ablate basal proliferative activity induced by lung injury (Figure 4, B and C), suggesting the presence of an Akt-independent pathway that complements MDM2-induced DNA replication in progenitor cells. This observation is consistent with a requirement of injury-induced signaling for progenitor cell proliferation. Our findings suggest that lung injury in individuals expressing more than normal levels of MDM2, either due to gene amplification or snp at the MDM2 promoter, could — in principle - activate progenitor cell proliferation and induce EMT. Under such conditions, inhibition of Akt signaling could be clinically important to restrict deregulated progenitor cell proliferation due to MDM2 overexpression consequent to lung injury.

Since destabilization of WT p53 by MDM2 may cause deregulated S phase entry, we compared DNA replication and reepithelialization after naphthalene injury in lungs of WT and p53-null mice. Our data (Supplemental Figure 3, C-H) did not show any appreciable statistically significant acceleration of injury repair in p53-null mice. These results are consistent with our earlier report that p53-null lung cells show deregulated $\mathrm{S}$ phase entry but reduced ability to complete genome duplication (54). Thus, p53-depletion does not serve as a surrogate for MDM2 in repair of lung injury. Using a mutant p53 knock-in mouse model with Dox-inducible MDM2 expression, we show that the ability of MDM2 to initiate DNA replication and progenitor cell proliferation after lung injury does not require the presence of WT p53, indicating that the MDM2-induced acceleration of reepithelialization is not a result of release from growth suppression by WT p53 due to its destabilization by MDM2 (Figure 7). In addition, we found that MDM2 can induce DNA replication in lung progenitor cells after naphthalene injury, while loss of the MDM2 allele in p53null mice abrogates this ability. These data indicate that MDM2 induces DNA replication specifically in lung progenitor cells, leading to their expansion to regenerate the lost epithelial layer after lung injury independently of WT p53. This unique property of MDM2 is essential for epithelial regeneration after lung injury, distinct from its ability to prevent WT p53-mediated apoptosis and cell death, which may reduce 
extent of injury $(18,55,56)$. Our data not only reveal a potentially novel role of MDM2 in repair of lung injury (Figure 10), but also show a cooperation between the oncoprotein MDM2 and proliferation of lung progenitor cells in the aftermath of lung injury.

\section{Methods}

Animal studies. pCMV tetracycline response element-tightMDM2 (pTRE-TightMDM2) mice were generated using C57BL/6 mice with the help of Institutional Transgenics Core. CCSPrtTA mice were a gift from Jeffery Whitsett, Cincinnati Children's Hospital (Cincinnati, Ohio, USA) (31). SPC-rtTA and tetO-Cre mice were purchased from the Jackson Laboratory (31, 57). p53 Lox-Stop-Lox R172H (p53 LSL R172H) knock-in mice were purchased from NCI Mouse Repository (58). p53 $3^{+/}: \mathrm{Mdm} 2^{+/-}$mice were a gift from Guillermina Lozano, M.D. Anderson Cancer Center, TX (13). pTRE-TightMDM2 mice were crossed with CCSPrtTA or SPC-rtTA mice to generate a litter of mixed genetic background mice and to allow inducible expression of human MDM2 in Club or the ATII cells of the mice lung. To direct p53-R172H and MDM2 expression to Club cells, LSL R172H mice were crossbred with pTRE-TightMDM2, tetO-Cre, and CCSPrtTA mice to produce the desired strain of mice. Both Cre recombinase and MDM2 expression was driven from promoters containing TRE and were activated following expression of rtTA from CCSP promoter and binding of rtTA to TRE in the presence of Dox effector. MDM2 expression and/or p53-R172H expression in these mice were induced by feeding them $500 \mu \mathrm{g} / \mathrm{ml}$ Dox in $5 \% \mathrm{w} / \mathrm{v}$ sucrose drinking water. Littermate mice on 5\% w/v sucrose drinking water (-Dox) and CCSPrtTA or SPC-rtTA only mice on Dox (+Dox) were used as controls.

Cell lines. $\mathrm{p} 53^{+/-}: \mathrm{MDM} 2$ transgenic and $\mathrm{p} 53^{+/-}$cells were generated by isolating lung cells from respective mice as described previously (37).

Drug treatments. For inducing injury in Club cells, naphthalene (MilliporeSigma) was dissolved in corn oil at $25 \mathrm{mg} / \mathrm{ml}$ concentration and administered i.p. at a dosage of $250 \mathrm{mg} / \mathrm{kg}$. Lungs were harvested 72 hours after injury. For proliferation studies, mice were given i.p. injections of $100 \mathrm{mg} / \mathrm{kg} \mathrm{BrdU}$ (MilliporeSigma) dissolved in sterile PBS 5 hours before sacrifice. For Akt inhibitor studies, MK-2206 HCl (Selleck Chemicals) was dissolved in 30\% Captisol (Thermo Fisher Scientific) and administered orally at a dose of $120 \mathrm{mg} / \mathrm{kg}$.

Irradiation protocol. CT-based treatment planning and execution used the Small Animal Radiation Research Platform (SARRP) from Xstrahl. Animals were anesthetized with 2\% isofluorane during CT-scanning and radiotherapy. Three beams were generated after CT, delivering a total dose of $14 \mathrm{~Gy}$ to the left lobe of the lung (59). The mice were euthanized, and lungs were harvested 3 months after the irradiation.

IHC and fluorescence. All staining was performed on paraffin embedded sections. Samples were fixed in $4 \%$ paraformaldehyde for at least 24 hours. Tissue embedding and sectioning was performed by Institutional Macromolecule Core Laboratory. IHC was performed with the Vectastain ABC Kit (Vector Labs) according to manufacturer's instructions and mounted using Permount solution (Thermo Fisher Scientific). BrdU incorporated sections were stained using BrdU staining kit (Invitrogen) following company's protocol. All counterstaining was performed using Hematoxylin solution (Vector Labs). IHC images were created using Labophot-2 microscope, captured using a 1080p HD 6MP microscope camera and processed using ISCapture software. Fluorescent stained sections were mounted using ProLong Gold Anitfade with DAPI and imaged by confocal microscopy (Zeiss LSM700) at $40 \times$ magnification. Average corrected fluorescence was measured using Image J software (NIH) (60).

Antibodies. Antibodies used included MDM2-N20, CCSP-T18, SP-C-FL197, p-GSK3ß-FL2 and p53FL393 (Santa-Cruz Biotechnology); p-H3 (9701), vimentin (5741), p- $\beta$-catenin Ser552 (5615) (Cell Signaling Technology); Ki-67 (AB-9260, MilliporeSigma); N-cadherin (PA5-29570, Thermo Fisher Scientific); and NICD (ab-8925, Abcam) according to manufacturer's protocol . For fluorescent immunostaining, BrdU was detected by mouse anti-bromodeoxyuridine (347580, Becton Dickinson) primary antibody. All fluorescent staining was performed using Alexa Fluor 488-conjugated chicken anti-goat (A21467), Alexa Fluor 594-conjugated donkey anti-rabbit IgG (A21207) and, Alexa Fluor 594-conjugated rabbit anti-mouse (A11062) secondary antibodies (Invitrogen) and mounted using ProLong Gold anti-fade reagent (P36930, Molecular Probes). Counter staining was performed using DAPI (MilliporeSigma).

$q P C R$. RNA was isolated from lung accessory lobes, and cDNA was synthesized using the Thermoscript Reverse Transcription-PCR system (Invitrogen). qPCR was carried out using a LightCycler system (Roche Diagnostics). Primers (Supplemental Table 1) were designed using OLIGO 5 software (Molecular 


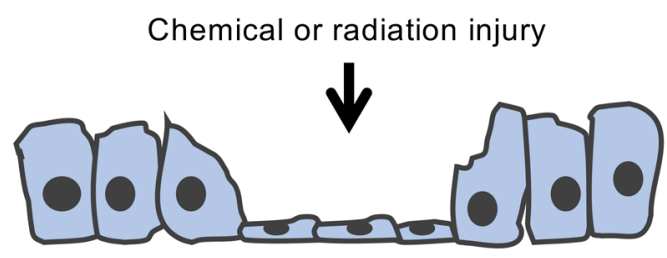

Depletion of epithelial cells

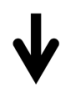

Release of growth factors

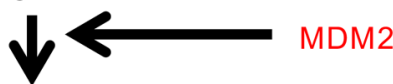

Activation of Akt signaling pathway

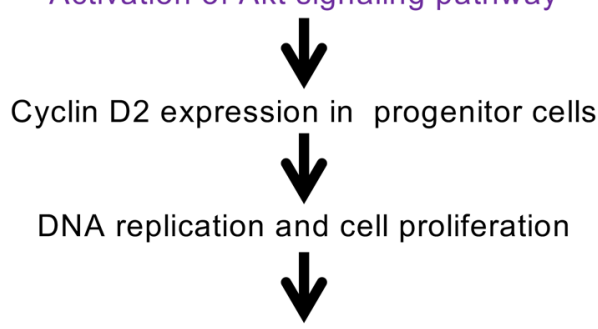

Epithelial mesenchymal transition

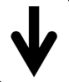

Epithelial regeneration
Figure 10. Proposed pathway for progenitor cell proliferation and repair of lung injury by MDM2. Consequence of lung injury in the context of MDM2 overexpression is depicted. MDM2-induced Akt phosphorylation accelerates DNA replication and proliferation of lung progenitor cells, restoring the epithelial layer depleted by injury.

Biology Insights) and were synthesized by Integrated DNA Technologies. Reactions were performed in triplicate utilizing SYBR green dye, which exhibits a higher fluorescence upon binding of double-stranded DNA. The methods have been described previously (54). Reactions were performed in triplicate and repeated in 3 different sets of mice.

Genotyping. Primers (Supplemental Table 2) used were as follows: (a) pTREtightMDM2: 5'-CTG TGA GTG AGA ACA GGT GTC AC-3' and 5'-GGC TAT AAT CTT CTG AGT CGA GAG-3'; (b) p53 $3^{+/ \text {: }}$ 5'-AGC GTG GTG GTA CCT TAT GAG C-3', 5'-GGA TGG TGG TAT ACT CAG AGC C-3', and 5'-GCT ATC AGG ACA TAG CGT TGG C-3'; and (c) MDM2+/-: 5'-TGT GGC TGG AGC ATG GGT ATT G-3', 5'-ATC TGA GAG CTC GTG CCC TTC G-3', and 5'-GGC GGA AAG AAC CAG CTG GGG C-3'.

Immunoblot analysis and quantification. Immunoblot analysis was performed following standard techniques. Antibodies are described above. Quantitative comparisons were performed using Quantity One 4.6.2 software (Bio-Rad).

siRNA transfection. siRNA transfection was performed using X-tremeGene siRNA transfection reagent (Roche Diagnostics) following manufacturer's protocol. A combination of mouse Akt1 siRNA (5'-AACCAGGACCACGAGAAGCUG-3') and mouse Akt2 siRNA (5'-GAGAGGACCUUCCAUGUAG-3') was used to silence Akt, and siControl (5'-TCTTAATCGCGTATAAGGC-3') was used as negative control.

Wound healing assay. The assay was performed according to manufacturer's instructions (Ibidi). Briefly, $3 \times$ $10^{5}$ mouse lung cells following siRNA transfection were seeded in the ibidi Culture-Inserts 2 Well. Once the cells reached confluency, the insert was removed, and fresh media was added to the plate and incubated at $37^{\circ} \mathrm{C}$. At 8 hours following insert removal, the gap-width of scratch was measured at 3 random fields and compared with the initial 0-hour gap size. A line was drawn to indicate the maximum distance traveled by a cell, and then the gap-width was measured using ImageJ software (NIH). The experiment was repeated 3 times.

Data availability. Data that support the findings of this study are available from the corresponding author upon request.

Statistics. For BrdU labeling and BASC quantification, total number of positive cells in 16 different bronchioles were calculated per mice. At least 3 different mice were used to calculate significance of differences between sample means using 1-tailed Student's $t$ test. All results are expressed as mean \pm SD. $P$ values less than 0.05 were considered statistically significant. Average corrected fluorescence and reepithe- 
lialization data was collected from blind images of 5 bronchioles in 3 different sets of mice. Results were plotted using a dot plot, and significance of differences between sample measurements was determined by Mann-Whitney-Wilcoxon test. $P$ values less than 0.05 were considered statistically significant. At least 3 mice of each genotype were used for each experiment.

Study approval. All animals used in this study were maintained and assayed in accordance with federal guidelines and those established by the Institutional Animal Care and Use Committee.

\section{Author contributions}

SS and CAV generated mouse constructs, while SS performed all the experiments involving immunostaining and RNA and protein analysis with and without injury. CR and RM designed and executed mice irradiation. SD contributed to experimental design. SPD conceived and designed experiments, supervised the work, and wrote the manuscript. All authors contributed to data analysis and preparation of the manuscript.

\section{Acknowledgments}

The authors thank Jeffery Whitsett, Cincinnati Children's Hospital, for his gift of CCSPrtTA mice, Guillermina Lozano for $\mathrm{p} 53^{+/-}: \mathrm{Mdm}^{+/-}$mice, and Lawrence Povirk, Virginia Commonwealth University, for insightful criticisms of the article. pTREtightMDM2 transgenic mice was generated by VCU transgenic core. Tissue sections were generated by the Virginia Commonwealth University Cancer Mouse Models Core Laboratory. This study was supported by institutional bridge funding and Massey Cancer Center pilot projects to SPD and SD and NCI R21-CA212967-01 to SD. Shilpa Singh was supported by VCU ILS program.

Address correspondence to: Swati Palit Deb, Department of Biochemistry and Molecular Biology, Virginia Commonwealth University, 401 College street, Room 311, Richmond, Virginia 23298, USA. Phone: 804.828.9541; Email: swati.deb@vcuhealth.org.

1. Karni-Schmidt O, Lokshin M, Prives C. The Roles of MDM2 and MDMX in Cancer. Annu Rev Pathol. 2016;11:617-644.

2. Vaughan C, et al. Human Oncoprotein MDM2 Up-regulates Expression of NF-кB2 Precursor p100 Conferring a Survival Advantage to Lung Cells. Genes Cancer. 2011;2(10):943-955.

3. Ding L, et al. Somatic mutations affect key pathways in lung adenocarcinoma. Nature. 2008;455(7216):1069-1075.

4. Senturk E, Manfredi JJ. Mdm2 and tumorigenesis: evolving theories and unsolved mysteries. Genes Cancer. 2012;3(3-4):192-198.

5. Bond GL, et al. A single nucleotide polymorphism in the MDM2 promoter attenuates the p53 tumor suppressor pathway and accelerates tumor formation in humans. Cell. 2004;119(5):591-602.

6. Bond GL, Hu W, Levine A. A single nucleotide polymorphism in the MDM2 gene: from a molecular and cellular explanation to clinical effect. Cancer Res. 2005;65(13):5481-5484.

7. Deben C, Deschoolmeester V, Lardon F, Rolfo C, Pauwels P. TP53 and MDM2 genetic alterations in non-small cell lung cancer Evaluating their prognostic and predictive value. Crit Rev Oncol Hematol. 2016;99:63-73.

8. Liu G, et al. Genetic polymorphisms of MDM2, cumulative cigarette smoking and nonsmall cell lung cancer risk. Int J Cancer. 2008;122(4):915-918

9. Altmayer NC, Galata V, Warschburger N, Keller A, Meese E, Fischer U. Gene amplification in mesenchymal stem cells and during differentiation towards adipocytes or osteoblasts. Oncotarget. 2018;9(2):1803-1812.

10. Lind H, Zienolddiny S, Ekstrøm PO, Skaug V, Haugen A. Association of a functional polymorphism in the promoter of the MDM2 gene with risk of nonsmall cell lung cancer. Int J Cancer. 2006;119(3):718-721.

11. Moyer SM, Larsson CA, Lozano G. Mdm proteins: critical regulators of embry ogenesis and homeostasis. J Mol Cell Biol. 2017;9(1):16-25.

12. Jones SN, Roe AE, Donehower LA, Bradley A. Rescue of embryonic lethality in Mdm2-deficient mice by absence of p53. Nature. 1995;378(6553):206-208.

13. Montes de Oca Luna R, Wagner DS, Lozano G. Rescue of early embryonic lethality in mdm2-deficient mice by deletion of p53. Nature. 1995;378(6553):203-206.

14. Jones SN, Hancock AR, Vogel H, Donehower LA, Bradley A. Overexpression of Mdm2 in mice reveals a p53-independent role for Mdm2 in tumorigenesis. Proc Natl Acad Sci USA. 1998;95(26):15608-15612.

15. Lundgren $\mathrm{K}$, et al. Targeted expression of MDM2 uncouples S phase from mitosis and inhibits mammary gland development independent of p53. Genes Dev. 1997;11(6):714-725.

16. Ganguli G, Abecassis J, Wasylyk B. MDM2 induces hyperplasia and premalignant lesions when expressed in the basal layer of the epidermis. EMBO J. 2000;19(19):5135-5147.

17. Folberg-Blum A, Sapir A, Shilo BZ, Oren M. Overexpression of mouse Mdm2 induces developmental phenotypes in Drosophila. Oncogene. 2002;21(15):2413-2417.

18. Hilliard SA, Yao X, El-Dahr SS. Mdm2 is required for maintenance of the nephrogenic niche. Dev Biol. 2014;387(1):1-14.

19. Mulay SR, Thomasova D, Ryu M, Anders HJ. MDM2 (murine double minute-2) links inflammation and tubular cell healing during acute kidney injury in mice. Kidney Int. 2012;81(12):1199-1211.

20. Wienken M, et al. MDM2 Associates with Polycomb Repressor Complex 2 and Enhances Stemness-Promoting Chromatin 
Modifications Independent of p53. Mol Cell. 2016;61(1):68-83.

21. Gorgoulis VG, et al. Altered expression of the cell cycle regulatory molecules pRb, p53 and MDM2 exert a synergetic effect on tumor growth and chromosomal instability in non-small cell lung carcinomas (NSCLCs). Mol Med. 2000;6(3):208-237.

22. Manfredi JJ. The Mdm2-p53 relationship evolves: Mdm2 swings both ways as an oncogene and a tumor suppressor. Genes Dev. 2010;24(15):1580-1589.

23. Kotton DN, Morrisey EE. Lung regeneration: mechanisms, applications and emerging stem cell populations. Nat Med. 2014;20(8):822-832.

24. Liu X, Driskell RR, Engelhardt JF. Stem cells in the lung. Meth Enzymol. 2006;419:285-321.

25. Reynolds SD, Malkinson AM. Clara cell: progenitor for the bronchiolar epithelium. Int J Biochem Cell Biol. 2010;42(1):1-4.

26. Wynn TA. Integrating mechanisms of pulmonary fibrosis. J Exp Med. 2011;208(7):1339-1350.

27. Königshoff M. Lung cancer in pulmonary fibrosis: tales of epithelial cell plasticity. Respiration. 2011;81(5):353-358.

28. Daniels CE, Jett JR. Does interstitial lung disease predispose to lung cancer? Curr Opin Pulm Med. 2005;11(5):431-437.

29. Brown DR, Thomas CA, Deb SP. The human oncoprotein MDM2 arrests the cell cycle: elimination of its cell-cycle-inhibitory function induces tumorigenesis. EMBO J. 1998;17(9):2513-2525.

30. Oliner JD, Pietenpol JA, Thiagalingam S, Gyuris J, Kinzler KW, Vogelstein B. Oncoprotein MDM2 conceals the activation domain of tumour suppressor p53. Nature. 1993;362(6423):857-860.

31. Tichelaar JW, Lu W, Whitsett JA. Conditional expression of fibroblast growth factor-7 in the developing and mature lung. $J$ Biol Chem. 2000;275(16):11858-11864

32. Pardee AB. A restriction point for control of normal animal cell proliferation. Proc Natl Acad Sci USA. 1974;71(4):1286-1290

33. Sobecki M, et al. Cell-Cycle Regulation Accounts for Variability in Ki-67 Expression Levels. Cancer Res. 2017;77(10):2722-2734.

34. Banerjee T, Chakravarti D. A peek into the complex realm of histone phosphorylation. Mol Cell Biol. 2011;31(24):4858-4873.

35. Hogan BL, et al. Repair and regeneration of the respiratory system: complexity, plasticity, and mechanisms of lung stem cell function. Cell Stem Cell. 2014;15(2):123-138.

36. Volckaert T, et al. Parabronchial smooth muscle constitutes an airway epithelial stem cell niche in the mouse lung after injury. $J$ Clin Invest. 2011;121(11):4409-4419.

37. Frum RA, et al. The human oncoprotein MDM2 induces replication stress eliciting early intra-S-phase checkpoint response and inhibition of DNA replication origin firing. Nucleic Acids Res. 2014;42(2):926-940.

38. Singh S, Ramamoorthy M, Vaughan C, Yeudall WA, Deb S, Palit Deb S. Human oncoprotein MDM2 activates the Akt signaling pathway through an interaction with the repressor element-1 silencing transcription factor conferring a survival advantage to cancer cells. Cell Death Differ. 2013;20(4):558-566.

39. Feng J, et al. Stabilization of Mdm2 via decreased ubiquitination is mediated by protein kinase B/Akt-dependent phosphorylation. J Biol Chem. 2004;279(34):35510-35517.

40. Mayo LD, Donner DB. A phosphatidylinositol 3-kinase/Akt pathway promotes translocation of Mdm2 from the cytoplasm to the nucleus. Proc Natl Acad Sci USA. 2001;98(20):11598-11603.

41. Astle MV, et al. AKT induces senescence in human cells via mTORC1 and p53 in the absence of DNA damage: implications for targeting mTOR during malignancy. Oncogene. 2012;31(15):1949-1962.

42. Xing Y, Li A, Borok Z, Li C, Minoo P. NOTCH1 is required for regeneration of Clara cells during repair of airway injury. Stem Cells. 2012;30(5):946-955.

43. McCubrey JA, et al. Effects of mutations in Wnt/ $\beta$-catenin, hedgehog, Notch and PI3K pathways on GSK-3 activity-Diverse effects on cell growth, metabolism and cancer. Biochim Biophys Acta. 2016;1863(12):2942-2976.

44. Gorgoulis VG, et al. Alterations of the p16-pRb pathway and the chromosome locus 9p21-22 in non-small-cell lung carcinomas: relationship with p53 and MDM2 protein expression. Am J Pathol. 1998;153(6):1749-1765.

45. Coggle JE, Lambert BE, Moores SR. Radiation effects in the lung. Environ Health Perspect. 1986;70:261-291.

46. Moding EJ, et al. An extra copy of $p 53$ suppresses development of spontaneous Kras-driven but not radiation-induced cancer. JCI Insight. 2016;1(10):e86698.

47. Midgley CA, Owens B, Briscoe CV, Thomas DB, Lane DP, Hall PA. Coupling between gamma irradiation, p53 induction and the apoptotic response depends upon cell type in vivo. J Cell Sci. 1995;108(Pt 5):1843-1848.

48. Kim CF, et al. Identification of bronchioalveolar stem cells in normal lung and lung cancer. Cell. 2005;121(6):823-835.

49. Crosby LM, Waters CM. Epithelial repair mechanisms in the lung. Am J Physiol Lung Cell Mol Physiol. 2010;298(6):L715-L731.

50. Araki S, et al. TGF-beta1-induced expression of human Mdm2 correlates with late-stage metastatic breast cancer. J Clin Invest. 2010;120(1):290-302.

51. Jung CR, et al. Enigma negatively regulates p53 through MDM2 and promotes tumor cell survival in mice. J Clin Invest. 2010;120(12):4493-4506.

52. Zhang K, et al. Amplification of FRS2 and activation of FGFR/FRS2 signaling pathway in high-grade liposarcoma. Cancer Res. 2013;73(4):1298-1307.

53. Rössig L, Jadidi AS, Urbich C, Badorff C, Zeiher AM, Dimmeler S. Akt-dependent phosphorylation of p21(Cip1) regulates PCNA binding and proliferation of endothelial cells. Mol Cell Biol. 2001;21(16):5644-5657.

54. Singh S, Vaughan CA, Frum RA, Grossman SR, Deb S, Palit Deb S. Mutant p53 establishes targetable tumor dependency by promoting unscheduled replication. J Clin Invest. 2017;127(5):1839-1855.

55. Gannon HS, Donehower LA, Lyle S, Jones SN. Mdm2-p53 signaling regulates epidermal stem cell senescence and premature aging phenotypes in mouse skin. Dev Biol. 2011;353(1):1-9.

56. Abbas HA, et al. Mdm2 is required for survival of hematopoietic stem cells/progenitors via dampening of ROS-induced p53 activity. Cell Stem Cell. 2010;7(5):606-617.

57. Perl AK, Wert SE, Nagy A, Lobe CG, Whitsett JA. Early restriction of peripheral and proximal cell lineages during formation of the lung. Proc Natl Acad Sci USA. 2002;99(16):10482-10487.

58. Olive KP, et al. Mutant p53 gain of function in two mouse models of Li-Fraumeni syndrome. Cell. 2004;119(6):847-860.

59. Jackson IL, et al. Characterization of the dose response relationship for lung injury following acute radiation exposure in three well-established murine strains: developing an interspecies bridge to link animal models with human lung. Health Phys. 
2014;106(1):48-55.

60. Burgess A, Vigneron S, Brioudes E, Labbé JC, Lorca T, Castro A. Loss of human Greatwall results in G2 arrest and multiple mitotic defects due to deregulation of the cyclin B-Cdc2/PP2A balance. Proc Natl Acad Sci USA. 2010;107(28):12564-12569. 